\title{
Theory of acoustic trapping of microparticles in capillary tubes
}

\author{
Bach, Jacob Søberg; Bruus, Henrik
}

Published in:

Physical Review E

Link to article, DOI:

10.1103/physreve.101.023107

Publication date:

2020

Document Version

Publisher's PDF, also known as Version of record

Link back to DTU Orbit

Citation (APA):

Bach, J. S., \& Bruus, H. (2020). Theory of acoustic trapping of microparticles in capillary tubes. Physical Review E, 101(2), [023107]. https://doi.org/10.1103/physreve.101.023107

\section{General rights}

Copyright and moral rights for the publications made accessible in the public portal are retained by the authors and/or other copyright owners and it is a condition of accessing publications that users recognise and abide by the legal requirements associated with these rights.

- Users may download and print one copy of any publication from the public portal for the purpose of private study or research.

- You may not further distribute the material or use it for any profit-making activity or commercial gain

- You may freely distribute the URL identifying the publication in the public portal

If you believe that this document breaches copyright please contact us providing details, and we will remove access to the work immediately and investigate your claim. 


\title{
Theory of acoustic trapping of microparticles in capillary tubes
}

\author{
Jacob S. Bach* and Henrik Bruus $\odot^{\dagger}$ \\ Department of Physics, Technical University of Denmark, and DTU Physics Building 309, DK-2800 Kongens Lyngby, Denmark
}

(Received 29 November 2019; accepted 5 February 2020; published 20 February 2020)

\begin{abstract}
We present a semianalytical theory for the acoustic fields and particle-trapping forces in a viscous fluid inside a capillary tube with arbitrary cross section and ultrasound actuation at the walls. We find that the acoustic fields vary axially on a length scale proportional to the square root of the quality factor of the two-dimensional (2D) cross-section resonance mode. This axial variation is determined analytically based on the numerical solution to the eigenvalue problem in the $2 \mathrm{D}$ cross section. The analysis is developed in two steps: First, we generalize a recently published expression for the $2 \mathrm{D}$ standing-wave resonance modes in a rectangular cross section to arbitrary shapes, including the viscous boundary layer. Second, based on these 2D modes, we derive analytical expressions in three dimensions for the acoustic pressure, the acoustic radiation and trapping force, as well as the acoustic energy flux density. We validate the theory by comparison to three-dimensional numerical simulations.
\end{abstract}

DOI: 10.1103/PhysRevE.101.023107

\section{INTRODUCTION}

Acoustophoresis is the acoustically induced migration of particles. During the past few decades the scientific field of microscale acoustofluidics has emerged, where this phenomenon is exploited for controlled handling of microparticles. Microscale acoustophoresis is gentle, label-free, and contact-less, and therefore it is useful for bioanalytics in lab-on-a-chip technologies. Examples include particle separation [1-4], concentration of red blood cells [5], iso-acoustic focusing of cells [6], acoustic tweezing [7-9], and cell patterning $[10,11]$. One particularly prominent acoustofluidic application is acoustic trapping of suspended microparticles against an external flow in cheap, disposable glass capillary tubes [12-17], which has been used for fast biological assays $[18,19]$ and for trapping of sub-micrometer particles by use of larger trapped seed particles [20,21]. In these systems, a piezoelectric transducer is attached to the capillary tube and driven at $\mathrm{MHz}$ frequencies to generate a standing-wave resonance mode localized inside the capillary tube above the transducer.

The physics behind acoustophoresis is primarily described by two time-averaged forces acting on the suspended particles. First, due to differences in density and compressibility between the particles and the carrier fluid, the particles experience the acoustic radiation force, which scales with the particle volume [22-27] and tends to focus particles. Second, due to time-averaged momentum fluxes induced by the acoustic fields, a steady acoustic streaming flow is generated, and suspended particles therefore experience a drag force, which scales with the particle radius and tends to mix particles [28-30].

Both the acoustic radiation force and the acoustic streaming are important in the acoustic trap. Aside from the

\footnotetext{
*jasoba@fysik.dtu.dk

†bruus@fysik.dtu.dk
}

one-dimensional (1D) or two-dimensional (2D) focusing in the cross section due to the transverse acoustic radiation force, the axial variations in the acoustic fields also give rise to an axial acoustic radiation force, or trapping force. Furthermore, acoustic streaming in the plane parallel to the transducer surface is often observed above the edges of the transducer, strongly affecting the trapping characteristics $[15,20]$. The acoustic trapping in capillaries is therefore a threedimensional (3D) problem, see Fig. 1, which complicates both the experimental characterization and the theoretical analysis needed for further development. Further complications arise from the many different $2 \mathrm{D}$ cross-sectional shapes that have been employed in the experimental studies of acoustofluidics in capillary channels: rectangular and trapezoidal shapes in etched silicon devices [31], flat channels with quarter-circleshaped or tapered side walls in wet-etched glass devices [32], circular shapes in glass tubes [17], rectangular shapes of different aspect ratios in glass tubes such as 1:1 [16], 1:10 [14], and 1:20 [14,33], and flat glass tubes with a bulging lid [14]. In addition, given the technique of pulling structured multimaterial fibers recently applied in microfluidics [34], practically any cross-sectional shape of capillary tubes for acoustofluidics can now be fabricated. This multitude of shapes needs to be addressed theoretically.

In this work, we present a method to semianalytically calculate the 3D acoustic pressure in a capillary tube of arbitrary cross section actuated in an axially confined region of length $L_{\text {act }}$ at the walls, see Fig. 1. Based on the either analytical or numerical solution to the $2 \mathrm{D}$ eigenvalue problem in the cross section, we derive analytical expressions in three dimensions for the acoustic pressure, the acoustic radiation force, the acoustic energy flux density, and the ratio between the axial and transverse acoustic trapping force. In particular, we show that for a $2 \mathrm{D}$ resonance mode $\alpha$, the axial component of the radiation force is proportional to $\sqrt{\bar{\Gamma}^{\alpha}}$, where $\bar{\Gamma}^{\alpha}$ is the damping coefficient of the resonance mode $\alpha$. In the special case of a 1D standing pressure wave in the cross 


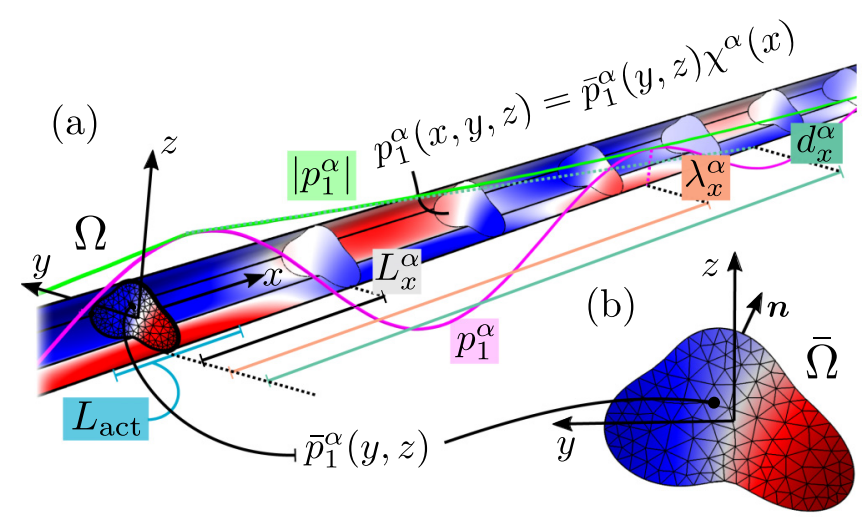

FIG. 1. A computed pressure resonance mode (dark red and light blue for high and low pressure) in a capillary tube of arbitrary cross section. (a) The complex-valued pressure mode $p_{1}^{\alpha}(x, y, z)$ in the 3D tube $\Omega$ is the product of the 2D pressure mode $\bar{p}_{1}^{\alpha}(y, z)$ in the cross section $\bar{\Omega}$ and the axial dependency $\chi^{\alpha}(x)$. It is excited by an actuation confined to a region of length $L_{\text {act }}$. The dark magenta and light green curves represent the real part of $p_{1}^{\alpha}$ and the magnitude $\left|p_{1}^{\alpha}\right|$, respectively. The relevant length scales in the $x$ direction are shown: the wave length $\lambda_{x}^{\alpha}$, the decay length $d_{x}^{\alpha}$, and the characteristic length scale $L_{x}^{\alpha}$; see Eqs. (20) and (21). (b) The pressure $\bar{p}_{1}^{\alpha}(y, z)$ in the 2D cross section $\bar{\Omega}$ with the surface normal vector $\boldsymbol{n}$ and the mesh used in the numerical simulations.

section, our results agree with Woodside et al. [35], who obtained an analytical expression for the axial radiation force being proportional to the axial gradient of the acoustic energy density $E_{\mathrm{ac}}$. However, whereas they left $E_{\mathrm{ac}}$ undetermined, we calculate it analytically.

We validate our analytical results by direct 3D numerical simulations. Recent contributions in the 3D numerical modeling of capillary tubes include Gralinski et al. [17] who modeled a circular capillary tube with fluid and glass, Lei et al. [15], who modeled the fluid domain of a capillary tube and found four in-plane streaming rolls, and Ley and Bruus [36], who took into account absorption of outgoing waves in both the glass and the fluid. Also the piezoelectric transducer may be included in a full-device simulation as done by Skov et al. [37]. In the numerical validation of this paper, we model the fluid domain with a prescribed movement of the fluid-solid interface and implement a perfectly matched layer (PML) to absorb outgoing waves.

The paper is organized as follows: We present the governing equations in Sec. II, and in Sec. III we describe the numerical implementation used for validation of the presented theory. In Sec. IV, we generalize our previous analytical results for the acoustic pressure in rectangular cross sections [38] to arbitrary cross sections. Using the residue theorem, we derive the axial dependency of the 3D acoustic pressure. We proceed in Sec. V by calculating the axial dependency of the pressure in the case of a box-shaped actuation, and in Sec. VC we validate the analytical results by $3 \mathrm{D}$ numerical simulations. In Sec. VI, we present analytical expressions for time-averaged acoustic quantities such as the axial radiation force and the axial energy flux density. Finally, we discuss our results in Sec. VII and conclude in Sec. VIII.

\section{GOVERNING EQUATIONS}

The physical displacement $\boldsymbol{u}_{\mathrm{phys}}^{0}(\boldsymbol{r}, t)$ of the fluid-solid interface oscillates harmonically with the angular frequency $\omega=2 \pi f$ and induces the physical pressure field $p_{\text {phys }}(\boldsymbol{r}, t)$ in the fluid. These fields are represented as the real part of the complex-valued linear perturbations $\boldsymbol{u}_{1}^{0}$ and $p_{1}$,

$$
\begin{aligned}
& \boldsymbol{u}_{\mathrm{phys}}^{0}(\boldsymbol{r}, t)=\operatorname{Re}\left[\boldsymbol{u}_{1}^{0}(\boldsymbol{r}) e^{-i \omega t}\right], \\
& p_{\text {phys }}(\boldsymbol{r}, t)=\operatorname{Re}\left[p_{1}(\boldsymbol{r}) e^{-i \omega t}\right] .
\end{aligned}
$$

In a fluid of dynamic viscosity $\eta_{\mathrm{fl}}$, bulk viscosity $\eta_{\mathrm{fl}}^{\mathrm{b}}$, isentropic compressibility $\kappa_{\mathrm{fl}}$, and mass density $\rho_{\mathrm{fl}}$, the acoustic fields are characterized by the compressional wave number $k_{\mathrm{c}}$ with real part $k_{0}=\frac{\omega}{c_{\mathrm{f}}}$, the bulk damping coefficient $\Gamma_{\mathrm{fl}}$, the shear wave number $k_{\mathrm{s}}$, and the viscous boundary-layer width $\delta_{\mathrm{s}}$ [27,30,37],

$$
\begin{aligned}
k_{\mathrm{c}} & =\left(1+i \frac{1}{2} \Gamma_{\mathrm{fl}}\right) k_{0}, & \Gamma_{\mathrm{fl}} & =\left(\frac{4}{3}+\frac{\eta_{\mathrm{fl}}^{\mathrm{b}}}{\eta_{\mathrm{fl}}}\right) \eta_{\mathrm{fl}} \kappa_{\mathrm{fl}} \omega, \\
k_{\mathrm{s}} & =\frac{1+i}{\delta_{\mathrm{s}}}, & \delta_{\mathrm{s}} & =\sqrt{\frac{2 \eta_{\mathrm{fl}}}{\rho_{\mathrm{fl}} \omega}},
\end{aligned}
$$

where $i=\sqrt{-1}$ is the imaginary unit. In this work, we assume that the viscous boundary layer is much thinner than the acoustic wave length, as is the case in most acoustofluidic applications,

$$
k_{0} \delta_{\mathrm{s}} \ll 1, \quad \Gamma_{\mathrm{fl}}=\frac{1}{2}\left(\frac{4}{3}+\frac{\eta_{\mathrm{fl}}^{\mathrm{b}}}{\eta_{\mathrm{fl}}}\right)\left(k_{0} \delta_{\mathrm{s}}\right)^{2} \ll 1 .
$$

The acoustic pressure $p_{1}$ satisfies the Helmholtz equation with the compressional wave number $k_{\mathrm{c}}$ and with the boundarylayer boundary condition recently derived in Ref. [30], valid for walls having a curvature radius much larger than the viscous boundary layer width $\delta_{\mathrm{s}}$,

$$
\begin{aligned}
\nabla^{2} p_{1}+k_{\mathrm{c}}^{2} p_{1} & =0, \quad \boldsymbol{r} \in \Omega, \\
\mathcal{D}_{\perp} p_{1} & =k_{0}^{2} U_{1 \perp}(\boldsymbol{r}), \quad \boldsymbol{r} \in \partial \Omega \\
\mathcal{D}_{\perp} & =\partial_{\perp}+\frac{i}{k_{\mathrm{s}}}\left(k_{\mathrm{c}}^{2}+\partial_{\perp}^{2}\right), \quad \boldsymbol{r} \in \partial \Omega \\
U_{1 \perp}(\boldsymbol{r}) & =\frac{\rho_{\mathrm{fl}} c_{\mathrm{fl}}^{2}}{1-i \Gamma_{\mathrm{fl}}}\left(-\boldsymbol{n}-\frac{i}{k_{\mathrm{s}}} \nabla\right) \cdot \boldsymbol{u}_{1}^{0}, \quad \boldsymbol{r} \in \partial \Omega .
\end{aligned}
$$

Here, the subscript $\perp$ represents the inward direction $(-\boldsymbol{n})$ opposite to the outward-pointing normal vector $\boldsymbol{n}$, and $U_{1 \perp}(\boldsymbol{r})$ is the effective actuation function defined in terms of the physical interface displacement $\boldsymbol{u}_{1}^{0}$ of the fluid-solid interface $\partial \Omega$. Finally, we write the following standard time-averaged acoustic quantities, all defined in terms of the pressure $p_{1}$ : The acoustic potential energy density $E_{\text {pot }}$, the acoustic kinetic energy density $E_{\text {kin }}$, the acoustic mechanical energy density $E_{\mathrm{ac}}$, the acoustic radiation potential $U_{\text {rad }}$ for a suspended spherical particle of radius $a$, the acoustic radiation force $\boldsymbol{F}_{\mathrm{rad}}$, and the acoustic energy flux density $S_{\mathrm{ac}}$,

$$
\begin{aligned}
& E_{\mathrm{pot}}=\frac{1}{4} \kappa_{\mathrm{fl}}\left|p_{1}\right|^{2}, \quad E_{\mathrm{kin}}=\frac{1}{4} \kappa_{\mathrm{fl}} k_{0}^{-2}\left|\nabla p_{1}\right|^{2}, \\
& E_{\mathrm{ac}}=E_{\mathrm{pot}}+E_{\mathrm{kin}}, \quad \boldsymbol{S}_{\mathrm{ac}}=\frac{1}{2 \rho_{\mathrm{fl}} \omega} \operatorname{Im}\left(p_{1}^{*} \nabla p_{1}\right), \\
& \boldsymbol{F}_{\mathrm{rad}}=-\nabla U_{\mathrm{rad}}, \quad U_{\mathrm{rad}}=\frac{4}{3} \pi a^{3}\left[f_{0} E_{\mathrm{pot}}-\frac{3}{2} f_{1} E_{\mathrm{kin}}\right] \text {. }
\end{aligned}
$$


TABLE I. Parameters used in the numerical simulations of water as the fluid medium at $25^{\circ} \mathrm{C}$ [42]; see also Sec. V C.

\begin{tabular}{lccc}
\hline \hline Parameter & Symbol & Value & Unit \\
\hline Mass density & $\rho_{\mathrm{fl}}$ & 997.05 & $\mathrm{~kg} \mathrm{~m}^{-3}$ \\
Compressibility & $\kappa_{\mathrm{fl}}$ & 448 & $\mathrm{TPa}^{-1}$ \\
Speed of sound & $c_{\mathrm{fl}}$ & 1496.7 & $\mathrm{~m} \mathrm{~s}^{-1}$ \\
Dynamic viscosity & $\eta_{\mathrm{fl}}$ & 0.890 & $\mathrm{mPa} \mathrm{s}$ \\
Bulk viscosity & $\eta_{\mathrm{fl}}^{\mathrm{b}}$ & 2.485 & $\mathrm{mPa} \mathrm{s}$ \\
Actuation displacement & $d_{0}$ & 0.1 & $\mathrm{~nm}$ \\
Cross-section length & $L_{\mathrm{cr}}$ & 300 & $\mu \mathrm{m}$ \\
Axial domain length & $L_{x}^{\text {num }}$ & 5.46 & $\mathrm{~cm}$ \\
PML length & $L_{\mathrm{PML}}$ & 500 & $\mu \mathrm{mm}$ \\
PML strength & $K_{\mathrm{PML}}$ & 1000 & - \\
\hline \hline
\end{tabular}

Here, $f_{0}$ and $f_{1}$ are the monopole and dipole scattering coefficients that are real-valued because we consider particles with radius $a$ much larger than both the viscous and the thermal boundary-layer thickness [22,25-27]. Furthermore, in Eq. (5b) "Im" and “ $*$ " denotes imaginary part and complex conjugation, respectively.

\section{NUMERICAL VALIDATION METHOD}

Our key theoretical results for the pressure $p_{1}$, to be presented in Secs. IV and V, are validated by the following direct-numerical-simulation method. We use the weak form PDE module in COMSOL MULTIPHYSICS [39] as described in Refs. $[30,37,40]$, see also an example cOMSOL script in the supplemental material of Ref. [41].

This validation is carried out in both a $3 \mathrm{D}$ and a $2 \mathrm{D}$ version solving the harmonically-driven problem (4) using the COMSOL "Stationary study." Moreover, as explained in Sec. IV, a main result of this work is that we can express the $3 \mathrm{D}$ pressure in terms of $2 \mathrm{D}$ pressure eigenmodes and eigenvalues, which we compute numerically using the COMSOL "Eigenvalue study." In the numerical simulations we use Lagrangian shape functions of quartic order, and the parameters listed in Table I.

For the numeric validation, we choose a capillary with the generic cross section shown in Fig. 1. This cross section has a linear size of around $2 L_{\mathrm{cr}}$, and its boundary $\partial \bar{\Omega}$ is given by the arbitrarily chosen, smooth, wavy parametric curve in the $y z$ cross section, $[y(s), z(s)]$ with $s \in[0 ; 2 \pi]$, defined by $y(s)=$ $L_{\mathrm{cr}} h_{\mathrm{cr}}(s) \cos (s), z(s)=0.9 L_{\mathrm{cr}} h_{\mathrm{cr}}(s) \sin (s+0.2)$, and the radius function $h_{\mathrm{cr}}(s)=1+0.15 \sin (2 s+1.5)-0.2 \sin (3 s)$.

The mesh is chosen to resolve the pressure on the relevant length scales. It is created as a $2 \mathrm{D}$ triangular mesh in the cross section with mesh size $\frac{1}{3} L_{\mathrm{cr}}$ in the bulk and $\frac{1}{6} L_{\mathrm{cr}}$ at the boundary; see Fig. 1(b). The 3D mesh is generated by sweeping the $2 \mathrm{D}$ mesh along the axial direction with a separation distance of $\frac{1}{6} L_{x}^{\alpha}$, where $L_{x}^{\alpha}$ is the characteristic axial length scale of the pressure introduced in Sec. IV C and shown in Fig. 1(a). The mesh is validated by standard mesh convergence tests [36].

For the 3D modeling of the long capillary tube, we use symmetry considerations to halve the computational domain [36], and a perfectly matched layer (PML) placed at the tube end to suppress acoustic reflections there; see Sec. II C of Ref. [36].

This numerical implementation of the model leads to $2 \times 10^{3}$ degrees of freedom (DOF) for the 2D simulations and $4 \times 10^{5} \mathrm{DOF}$ for the $3 \mathrm{D}$ simulations. The simulations were performed on a workstation with a $3.5 \mathrm{GHz}$ Intel Xeon CPU E5-1650 v2 dual-core processor and with a memory of 128 GB RAM.

Finally, we use the $L^{2}$-norm to numerically compute the relative deviation $\mathcal{E}\left(p, p^{\text {ref }}\right)$ of a pressure field $p$ from a reference pressure field $p^{\text {ref }}$ in the $3 \mathrm{D}$ domain $\Omega$ as

$$
\mathcal{E}\left(p, p^{\mathrm{ref}}\right)=\sqrt{\frac{\int_{\Omega}\left|p-p^{\mathrm{ref}}\right|^{2} d V}{\int_{\Omega}\left|p^{\mathrm{ref}}\right|^{2} d V}} .
$$

The analogous relative deviation in the 2D domain $\bar{\Omega}$ is called $\overline{\mathcal{E}}\left(\bar{p}, \bar{p}^{\text {ref }}\right)$, where an overbar denote a $2 \mathrm{D}$ quantity.

\section{THE ACOUSTIC PRESSURE IN A LONG STRAIGHT CAPILLARY TUBE OF ARBITRARY CROSS SECTION}

In the following, we calculate the acoustic pressure $p_{1}(x, y, z)$ satisfying Eq. (4) to lowest order in the small parameters $k_{0} \delta_{\mathrm{s}}$, Eq. (3), in a long, straight capillary tube of arbitrary cross section that is invariant in the axial $x$ direction as shown in Fig. 1(a). Our strategy has two key steps: First, based on our previous analysis of the 2D cross-sectional resonance modes $\bar{p}_{1}^{m n}(x, y)$ in a rectangular cross section having integer $m$ and $n$ half-waves in the $y$ and $z$ direction, and including the viscous boundary layer [38], we write an expression for the $2 \mathrm{D}$ cross-sectional resonance modes $\bar{p}_{1}^{\alpha}(x, y)$ in an arbitrary cross section. Second, by using these 2D modes together with the residue theorem, we evaluate the $3 \mathrm{D}$ acoustic pressure $p_{1}(x, y, z)$ satisfying Eq. (4) for any frequency $f=\frac{1}{2 \pi} \omega$ and actuation function $U_{1 \perp}$ as a sum over all resonance modes $\alpha$. This approach is valuable because it provides a physical understanding of the acoustic trapping in the axial direction and analytical scaling laws for various length scales and trapping forces in terms of the properties of the $2 \mathrm{D}$ cross-sectional resonance modes for any given shape.

\section{A. The 2D pressure resonance modes in an arbitrary cross section}

In Ref. [38], we studied the special case of a rectangular cross section of side lengths $L_{y}$ and $L_{z}$. We derived to lowest order in the small parameter $k_{0} \delta_{\mathrm{s}}$, the resonance modes $\bar{p}_{1}^{m n}$ with $m$ half-waves in the $y$ direction and $n$ half-waves in the $z$ direction, valid for wave numbers $k_{0}$ close to the resonance wave number $\bar{k}_{0}^{m n}$. Here, and in the following, we use the overbar to denote a quantity defined in the cross section $\bar{\Omega}$. With this notation, the expression for $\bar{p}_{1}^{m n}$ given in Eq. (12) of Ref. [38] becomes

$$
\begin{aligned}
\bar{p}_{1}^{m n}\left(k_{0} ; y, z\right) & =\bar{P}_{1}^{m n} \overline{\mathcal{G}}^{m n}\left(k_{0}\right) \bar{R}^{m n}(y, z), \quad \text { for } k_{0} \approx \bar{k}_{0}^{m n}, \\
\bar{R}^{m n}(y, z) & =\cos \left(\frac{m \pi y}{L_{y}}\right) \cos \left(\frac{n \pi z}{L_{z}}\right), \\
\bar{P}_{1}^{m n} & =\frac{\oint_{\partial \bar{\Omega}} \bar{U}_{1 \perp} \bar{R}^{m n} d l}{\int_{\bar{\Omega}}\left(\bar{R}^{m n}\right)^{2} d A},
\end{aligned}
$$




$$
\overline{\mathcal{G}}^{m n}\left(k_{0}\right)=\frac{\frac{1}{2} \bar{k}_{0}^{m n}}{k_{0}-\bar{k}_{0}^{m n}+\frac{1}{2} i \bar{k}_{0}^{m n} \bar{\Gamma}^{m n}} .
$$

The quantities used here have the following meaning: $\bar{R}^{m n}(y, z)$ in Eq. (7b) is the spatial dependency of a given 2D cross-sectional resonance mode. $\bar{P}_{1}^{m n}$ in Eq. (7c) is a coupling coefficient related to the overlap between $\bar{R}^{m n}(y, z)$ and the actuation function $\bar{U}_{1 \perp}(y, z)$ defined in Eq. (4d) on the boundary $\partial \bar{\Omega}$ of the cross section $\bar{\Omega} . \overline{\mathcal{G}}^{m n}\left(k_{0}\right)$ in Eq. (7d) is the line-shape function of the mode defined in terms of three parameters: the wave number $k_{0}=\frac{\omega}{c_{\mathrm{fl}}}$, the resonance wave number $\bar{k}_{0}^{m n}$, and the minute damping coefficient $\bar{\Gamma}^{m n}=\bar{\Gamma}_{\mathrm{bl}}^{m n}+\Gamma_{\mathrm{fl}} \ll 1$, where the latter is defined in Eq. (10) of Ref. [38] as the sum of the boundary-layer damping coefficient $\bar{\Gamma}_{\mathrm{bl}}^{m n}$ and the bulk damping coefficient $\Gamma_{\mathrm{fl}}$ of Eq. (2a).

\section{Generalization to an arbitrarily shaped $2 D$ cross section}

To generalize from the rectangular cross section to an arbitrarily shaped cross section, it is helpful to write $\overline{\mathcal{G}}^{m n}$ as a function not of $k_{0}$ but of the complex-valued wave number $k_{\mathrm{c}}$ from Eq. (2a) for $k_{\mathrm{c}} \approx \bar{k}_{\mathrm{c}}^{m n}$, where $\bar{k}_{\mathrm{c}}^{m n}$ is the complex-valued resonance wave number. This variable shift is obtained by inserting $\bar{\Gamma}^{m n}=\bar{\Gamma}_{\mathrm{bl}}^{m n}+\Gamma_{\mathrm{fl}}$ in Eq. (7d),

$$
\overline{\mathcal{G}}^{m n}\left(k_{\mathrm{c}}\right) \approx \frac{\left(\bar{k}_{\mathrm{c}}^{m n}\right)^{2}}{k_{\mathrm{c}}^{2}-\left(\bar{k}_{\mathrm{c}}^{m n}\right)^{2}}, \quad \bar{k}_{\mathrm{c}}^{m n}=\left(1-i \frac{\bar{\Gamma}_{\mathrm{bl}}^{m n}}{2}\right) \bar{k}_{0}^{m n} .
$$

In Eqs. (7) and (8), we substitute the mode index $m n$ by $\alpha$, and thereby introduce our main assumption, which is validated numerically below, namely, an expression for the pressure resonance mode $\bar{p}_{1}^{\alpha}$ in an arbitrary cross section valid close to resonance $k_{\mathrm{c}} \approx \bar{k}_{\mathrm{c}}^{\alpha}$ and to lowest order in the small parameter $k_{0} \delta_{\mathrm{s}}$,

$$
\begin{aligned}
\bar{p}_{1}^{\alpha}\left(k_{\mathrm{c}} ; y, z\right) & =\bar{P}_{1}^{\alpha} \overline{\mathcal{G}}^{\alpha}\left(k_{\mathrm{c}}\right) \bar{R}^{\alpha}(y, z), \quad \text { for } k_{\mathrm{c}} \approx \bar{k}_{\mathrm{c}}^{\alpha}, \\
\bar{P}_{1}^{\alpha} & =\frac{\oint_{\partial \bar{\Omega}} \bar{U}_{1 \perp} \bar{R}^{\alpha} d l}{\int_{\bar{\Omega}}\left(\bar{R}^{\alpha}\right)^{2} d A}, \\
\overline{\mathcal{G}}^{\alpha}\left(k_{\mathrm{c}}\right) & =\frac{\left(\bar{k}_{\mathrm{c}}^{\alpha}\right)^{2}}{k_{\mathrm{c}}^{2}-\left(\bar{k}_{\mathrm{c}}^{\alpha}\right)^{2}}, \quad \bar{k}_{\mathrm{c}}^{\alpha}=\left(1-i \frac{\bar{\Gamma}_{\mathrm{bl}}^{\alpha}}{2}\right) \bar{k}_{0}^{\alpha} .
\end{aligned}
$$

Here, the eigenvalue $\bar{k}_{\mathrm{c}}^{\alpha}$ and eigenfunction $\bar{R}^{\alpha}$ are defined through the 2D eigenvalue problem, corresponding to Eq. (4), in the cross section $\bar{\Omega}$ without actuation,

$$
\begin{array}{rlrl}
\nabla^{2} \bar{R}^{\alpha}+\left(\bar{k}_{\mathrm{c}}^{\alpha}\right)^{2} \bar{R}^{\alpha} & =0, & & \boldsymbol{r} \in \bar{\Omega}, \\
\mathcal{D}_{\perp} \bar{R}^{\alpha} & =0, \quad r \in \partial \bar{\Omega} .
\end{array}
$$

The resonance frequency $\bar{f}^{\alpha}$ of the 2D mode $\alpha$ is found from the real part $\bar{k}_{0}^{\alpha}=\operatorname{Re}\left(\bar{k}_{\mathrm{c}}^{\alpha}\right)$ of the eigenvalue $\bar{k}_{\mathrm{c}}^{\alpha}$,

$$
\bar{f}^{\alpha}=\frac{1}{2 \pi} \bar{\omega}^{\alpha}=\frac{1}{2 \pi} c_{\mathrm{fl}} \bar{k}_{0}^{\alpha} .
$$

The damping coefficient $\bar{\Gamma}^{\alpha}$ of mode $\alpha$ is written as the sum of the bulk damping coefficient $\Gamma_{\mathrm{fl}}$ of Eq. (2a) and the boundarylayer damping coefficient $\bar{\Gamma}_{\mathrm{bl}}^{\alpha}$ of Eq. (9c),

$$
\bar{\Gamma}^{\alpha}=\bar{\Gamma}_{\mathrm{bl}}^{\alpha}+\Gamma_{\mathrm{fl}}, \quad \bar{\Gamma}_{\mathrm{bl}}^{\alpha}=-\frac{2 \operatorname{Im}\left(\bar{k}_{\mathrm{c}}^{\alpha}\right)}{\operatorname{Re}\left(\bar{k}_{\mathrm{c}}^{\alpha}\right)} .
$$

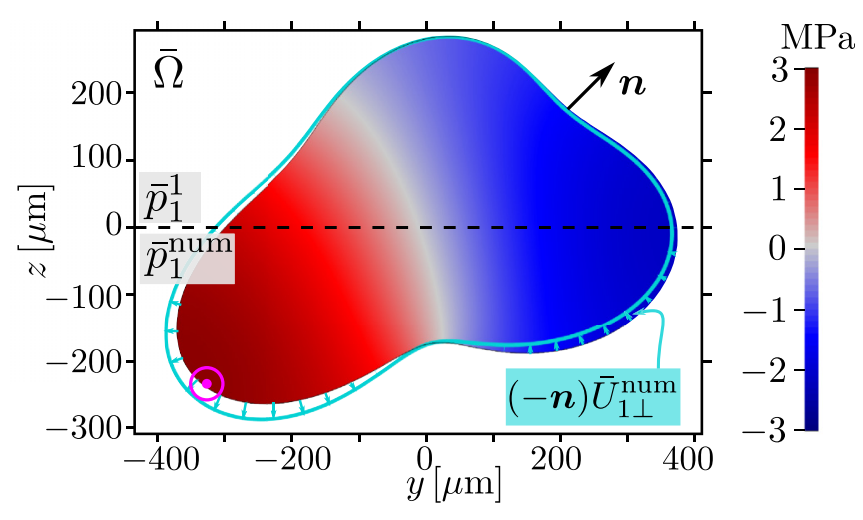

FIG. 2. Comparison in the cross section $\bar{\Omega}$ (defined in the text of Sec. III and shown in Fig. 1) between the 2D pressure mode $\bar{p}_{1}^{1}$ from Eqs. (9) and (10) with $\alpha=1$ plotted for $z>0$, and the 2D numerical pressure $\bar{p}_{1}^{\text {num }}$ from Eq. (4) plotted for $z<0$, both actuated by the actuation function $\bar{U}_{1 \perp}^{\text {num }}$ [light cyan curve and arrows, see Eq. (13)] at the fundamental resonance frequency $\bar{f}^{1}=1.1341 \mathrm{MHz}$. The relative deviation defined in Eq. (6) between $\bar{p}_{1}^{1}$ and $\bar{p}_{1}^{\text {num }}$ is $\overline{\mathcal{E}}\left(\bar{p}_{1}^{1}, \bar{p}_{1}^{\text {num }}\right)=0.14 \%$. The encircled magenta point marks the position of the line used in the 3D line plots of Sec. V C.

\section{Numerical validation in the $2 D$ cross section}

In Fig. 2, we validate numerically the generalized resonance-mode structure Eq. (9) by using the cross section $\bar{\Omega}$ and the numerical procedure described in Sec. III. We choose the actuation function $\bar{U}_{1 \perp}^{\text {num }}$ along the boundary $\partial \bar{\Omega}$ of $\bar{\Omega}$ to be

$$
\bar{U}_{1 \perp}^{\text {num }}(y, z)=\rho_{\mathrm{fl}} c_{\mathrm{fl}}^{2} d_{0} \sin \left(\frac{\pi}{L_{\mathrm{cr}}} y\right) e^{-\frac{z}{L_{\mathrm{cr}}}} .
$$

We determine numerically the lowest eigenmode $\alpha=1$ in terms of the eigenfunction $\bar{R}^{1}(y, z)$, Eq. (10), and eigenfrequency $\bar{f}^{1}$, Eq. (11), listed in Table II together with other relevant mode parameters for $\alpha=1$. Inserting this eigenmode together with $\bar{U}_{1 \perp}^{\text {num }}(y, z)$ and $k_{\mathrm{c}}=\frac{2 \pi}{c_{0}}\left(1+i \frac{1}{2} \Gamma_{\mathrm{fl}}\right) \bar{f}^{1}$ into Eq. (9), we compute the pressure resonance mode $\bar{p}_{1}^{1}\left(k_{\mathrm{c}} ; y, z\right)$ at the resonance frequency. In Fig. 2 we compare this theoretical result $\bar{p}_{1}^{1}$ with the direct numerical simulation $\bar{p}_{1}^{\text {num }}$ obtained from the 2D version of Eq. (4) at the resonance frequency $\bar{f}^{1}$. Qualitatively, we see a smooth transition passing from $\bar{p}_{1}^{1}$ above the dashed line $(z>0)$ to $\bar{p}_{1}^{\text {num }}$ below the dashed line $(z<0)$. Quantitatively, the relative difference $(6)$ between the semianalytical $\bar{p}_{1}^{1}$ and the numerical $\bar{p}_{1}^{\text {num }}$ is found to be $\overline{\mathcal{E}}\left(\bar{p}_{1}^{1}, \bar{p}_{1}^{\text {num }}\right)=0.14 \%$, which is satisfactory in this approximation to lowest order in the small boundary-layer-width parameter $k_{0} \delta_{\mathrm{s}}=0.24 \%$.

TABLE II. Values for the fundamental mode $\alpha=1$ of the 2D eigenvalue problem obtained by numerical simulation.

\begin{tabular}{lcccc}
\hline \hline Parameter & Symbol & Eq. & Value & Unit \\
\hline Eigenvalue & $\bar{k}_{\mathrm{c}}^{1}$ & Eq. (10) & $4761.01-3.49 i$ & $\mathrm{~m}^{-1}$ \\
Eigenfrequency & $\bar{f}^{1}$ & Eq. (11) & 1.1341 & $\mathrm{MHz}$ \\
Damping coefficient & $\bar{\Gamma}^{1}$ & Eq. (12) & 0.00148 & - \\
$x$ length scale & $L_{x}^{1}$ & Eq. (20) & 5.46 & $\mathrm{~mm}$ \\
\hline \hline
\end{tabular}




\section{B. The 3D pressure}

Based on Eq. (9) for the 2D cross-sectional pressure modes $\bar{p}_{1}^{\alpha}$, we now derive the pressure $p_{1}(x, y, z)$ satisfying Eq. (4) in the 3D capillary tube. For any given $x$-dependent function $\phi(x)$, we denote its Fourier transform by $\hat{\phi}\left(k_{x}\right)$, see Appendix A. The 3D pressure is calculated from the inverse Fourier transform,

$$
p_{1}(x, y, z)=\int_{-\infty}^{\infty} \hat{p}_{1}\left(k_{x} ; y, z\right) e^{i k_{x} x} \frac{d k_{x}}{2 \pi} .
$$

Since the integrand $\hat{p}_{1}\left(k_{x} ; y, z\right) e^{i k_{x} x}$ is a function of the complex-valued wave number $k_{x}$, we evaluate the integral using the residue theorem for an appropriate closed contour $\gamma$ in the complex $k_{x}$ plane and find

$$
p_{1}(x, y, z)=\sum_{k_{x}^{\alpha} \text { inside } \gamma} i \operatorname{Res}\left(\hat{p}_{1}\left(k_{x} ; y, z\right) e^{i k_{x} x}, k_{x}^{\alpha}\right),
$$

summing over the residues $\operatorname{Res}\left(\hat{p}_{1}\left(k_{x} ; y, z\right) e^{i k_{x} x}, k_{x}^{\alpha}\right)$ of all poles $k_{x}^{\alpha}$ inside the closed contour $\gamma$. To obtain these residues, we only need an expression for $\hat{p}_{1}\left(k_{x} ; y, z\right) e^{i k_{x} x}$ valid close to $k_{x}^{\alpha}$. The Fourier transform $\hat{p}_{1}\left(k_{x} ; y, z\right)$ satisfies the Fouriertransformed Helmholtz problem Eq. (4),

$$
\begin{aligned}
& \nabla^{2} \hat{p}_{1}\left(k_{x} ; y, z\right)+\left(k_{\mathrm{c}}^{2}-k_{x}^{2}\right) \hat{p}_{1}\left(k_{x} ; y, z\right)=0, \quad \boldsymbol{r} \in \bar{\Omega}, \\
& \mathcal{D}_{\perp} \hat{p}_{1}\left(k_{x} ; y, z\right)=k_{0}^{2} \hat{U}_{1 \perp}\left(k_{x} ; y, z\right), \quad \boldsymbol{r} \in \partial \bar{\Omega},
\end{aligned}
$$

where $\partial \bar{\Omega}$ is the boundary of $\bar{\Omega}$. We note that Eq. (15) for $\hat{p}_{1}\left(k_{x} ; y, z\right)$ is similar to Eq. (4) for an $x$-independent pressure $p_{1}=\bar{p}_{1}(y, z)$ with the substitutions $k_{\mathrm{c}}^{2} \rightarrow k_{\mathrm{c}}^{2}-k_{x}^{2}$ and $\bar{U}_{1 \perp}(y, z) \rightarrow \hat{U}_{1 \perp}\left(k_{x} ; y, z\right)$, see Appendix B for details. Using these substitutions in Eq. (9), we obtain the result for $\hat{p}_{1}\left(k_{x} ; y, z\right) e^{i k_{x} x}$ valid for $k_{\mathrm{c}}^{2}-k_{x}^{2} \approx\left(\bar{k}_{\mathrm{c}}^{\alpha}\right)^{2}$ and to lowest order in the small parameter $k_{0} \delta_{\mathrm{s}}$,

$$
\begin{gathered}
\hat{p}_{1}\left(k_{x} ; y, z\right) e^{i k_{x} x} \approx \frac{-\left(\bar{k}_{\mathrm{c}}^{\alpha}\right)^{2} \bar{R}^{\alpha} e^{i k_{x} x}}{\left(k_{x}\right)^{2}-\left(k_{x}^{\alpha}\right)^{2}} \frac{\int_{\partial \bar{\Omega}} \hat{U}_{1 \perp}\left(k_{x} ; y, z\right) \bar{R}^{\alpha} d l}{\int_{\bar{\Omega}}\left(\bar{R}^{\alpha}\right)^{2} d A}, \\
k_{x}^{\alpha}=\sqrt{k_{\mathrm{c}}^{2}-\left(\bar{k}_{\mathrm{c}}^{\alpha}\right)^{2}} .
\end{gathered}
$$

From Eq. (16a) we see that $\hat{p}_{1}\left(y, z, k_{x}\right) e^{i k_{x} x}$ has simple poles in the complex $k_{x}$ plane at $k_{x}= \pm k_{x}^{\alpha}$, and therefore the residues $\operatorname{Res}\left(\hat{p}_{1}\left(k_{x} ; y, z\right) e^{i k_{x} x}, k_{x}^{\alpha}\right)$ used in the sum Eq. (14b) can be found analytically; see Appendix C. The resulting expression for $p_{1}(x, y, z)$, valid for all frequencies and to lowest order in $k_{0} \delta_{\mathrm{s}}$, is

$$
\begin{aligned}
p_{1}(x, y, z) & =\sum_{\alpha} p_{1}^{\alpha}(x, y, z), \\
p_{1}^{\alpha}(x, y, z) & =P_{1}^{\alpha}(x) \overline{\mathcal{G}}^{\alpha}\left(k_{\mathrm{c}}\right) \bar{R}^{\alpha}(y, z), \\
P_{1}^{\alpha}(x) & =\left[\frac{\int_{\partial \bar{\Omega}} U_{1 \perp} \bar{R}^{\alpha} d l}{\int_{\bar{\Omega}}\left(\bar{R}^{\alpha}\right)^{2} d A} * g^{\alpha}\right](x), \\
g^{\alpha}(x) & =\frac{-i k_{x}^{\alpha}}{2} e^{i k_{x}^{\alpha}|x|} .
\end{aligned}
$$

Here, the asterisk " $*$ " denotes the usual functional convolution in the $x$ coordinate; see Eq. (A3). $g^{\alpha}(x)$ is the Green's function in the axial direction of mode $\alpha$ corresponding to a delta-function actuation at $x=0$, given by $U_{1 \perp}(x, y, z)=$ $\bar{U}_{1 \perp}(y, z) L_{\text {act }} \delta(x)$, as this actuation yields

$$
P_{1}^{\alpha}(x)=\bar{P}_{1}^{\alpha} L_{\mathrm{act}} g^{\alpha}(x),
$$

where $L_{\text {act }}$ is an actuation strength of dimension length, and $\bar{P}_{1}^{\alpha}$ is the 2D coupling coefficient defined in Eq. (7c).

\section{The axial length scales of each mode}

The axial dependency of the pressure $p_{1}(x, y, z)$ is given in Eqs. (17c) and (17d) by the actuation function $U_{1 \perp}$ and the Green's function $g^{\alpha}$. The latter leads to three axial length scales that characterize each mode $\alpha$. Here the strength of our approach become apparent, as it allows us to extract analytical expressions for these length scales. First, by using $k_{\mathrm{c}}^{2}=(1+$ $\left.i \Gamma_{\mathrm{fl}}\right) k_{0}^{2}$, Eq. (2a), and $\left(\bar{k}_{\mathrm{c}}^{\alpha}\right)^{2}=\left(1-i \bar{\Gamma}_{\mathrm{bl}}^{\alpha}\right)\left(\bar{k}_{0}^{\alpha}\right)^{2}$, Eq. (9c), as well as the assumption $\bar{\Gamma}^{\alpha} \ll 1$, we write the $x$ wave number $k_{x}^{\alpha}$ in Eq. (16b) of mode $\alpha$ as

$$
k_{x}^{\alpha}=\bar{k}_{\mathrm{c}}^{\alpha} \frac{1}{\sqrt{\overline{\mathcal{G}}^{\alpha}}} \approx \bar{k}_{0}^{\alpha} \sqrt{\bar{\Gamma}^{\alpha}} \sqrt{\Delta^{\alpha}+i}, \quad \Delta^{\alpha}=\frac{k_{0}^{2}-\left(\bar{k}_{0}^{\alpha}\right)^{2}}{\left(\bar{k}_{0}^{\alpha}\right)^{2} \bar{\Gamma}^{\alpha}} .
$$

Here, $\Delta^{\alpha}$ is the difference between the square of the wave number $k_{0}=\frac{\omega}{c_{\text {f }}}$ and the resonance wave number $\bar{k}_{0}^{\alpha}$, Eq. (11), of the mode $\alpha$ scaled by $\left(\bar{k}_{0}^{\alpha}\right)^{2} \bar{\Gamma}^{\alpha}$. From Eq. (19), we identify the characteristic length scale $L_{x}^{\alpha}$ of variation of the mode $\alpha$ in the $x$ direction as

$$
L_{x}^{\alpha}=\frac{1}{\bar{k}_{0}^{\alpha} \sqrt{\bar{\Gamma}^{\alpha}}} .
$$

Then, by using Eqs. (19) and (20), we write the decay length $d_{x}^{\alpha}$ and wave length $\lambda_{x}^{\alpha}$ of the function $g^{\alpha}(x)$ in Eq. (17d) as

$$
\begin{aligned}
& d_{x}^{\alpha}=\frac{1}{\operatorname{Im}\left(k_{x}^{\alpha}\right)}=L_{x}^{\alpha} \frac{1}{\operatorname{Im}\left(\sqrt{\Delta^{\alpha}+i}\right)}, \\
& \lambda_{x}^{\alpha}=2 \pi \frac{1}{\operatorname{Re}\left(k_{x}^{\alpha}\right)}=L_{x}^{\alpha} \frac{2 \pi}{\operatorname{Re}\left(\sqrt{\Delta^{\alpha}+i}\right)} .
\end{aligned}
$$

In the following, a tilde is used to denote rescaling by $L_{x}^{\alpha}$ in the axial direction,

$$
\begin{aligned}
\tilde{x} & =\frac{x}{L_{x}^{\alpha}}, \quad \tilde{d}_{x}^{\alpha}=\frac{d_{x}^{\alpha}}{L_{x}^{\alpha}}, \quad \tilde{\lambda}_{x}^{\alpha}=\frac{\lambda_{x}^{\alpha}}{L_{x}^{\alpha}}, \\
\tilde{k}_{x}^{\alpha} & =L_{x}^{\alpha} k_{x}^{\alpha}, \quad \tilde{g}^{\alpha}=L_{x}^{\alpha} g^{\alpha} .
\end{aligned}
$$

In Fig. 3(a), we plot the rescaled Green's function $\tilde{g}^{\alpha}(x)$ for three different frequencies: above resonance $\left(k_{0}=\bar{k}_{0}^{\alpha}+\right.$ $\left.\bar{k}_{0}^{\alpha} \bar{\Gamma}^{\alpha}, \Delta^{\alpha}=2\right)$, where it is propagating, at resonance $\left(k_{0}=\right.$ $\left.\bar{k}_{0}^{\alpha}, \Delta^{\alpha}=0\right)$, and below resonance $\left(k_{0}=\bar{k}_{0}^{\alpha}-\bar{k}_{0}^{\alpha} \bar{\Gamma}^{\alpha}, \Delta^{\alpha}=\right.$ -2 ), where it is evanescent. In Fig. 3(b), we plot the decay length $d_{x}^{\alpha}$ and the wave length $\lambda_{x}^{\alpha}$ of Eq. (21) as a function of the actuation frequency for frequencies close to resonance $k_{0} \approx \bar{k}_{0}^{\alpha}$. For frequencies just below resonance, $d_{x}^{\alpha}$ is small and $\lambda_{x}^{\alpha}$ is large, and vice versa for frequencies just above the resonance frequency. The three values of $\Delta^{\alpha}$ used in Fig. 3(a) are marked by dashed vertical lines of the same color in Fig. 3(b).

As an example of the characteristic length scale of the axial pressure variation $L_{x}^{\alpha}$, we consider a standing vertical half wave in a rectangular cross section of height $L_{z}$ with the 


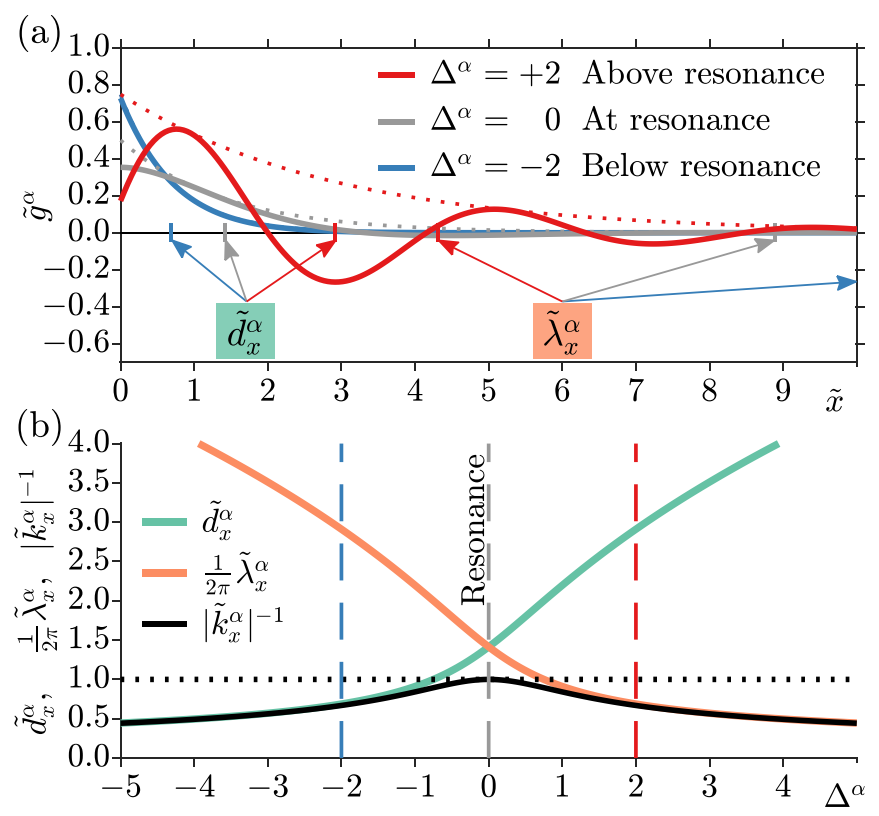

FIG. 3. Plots of the rescaled quantities of Eq. (22). (a) The complex-valued Green's function $\tilde{g}^{\alpha}(x)$ of Eq. (17d) plotted as the real part (solid) and modulus (dotted) for three values of $\Delta^{\alpha}$ of Eq. (19): Below (-2), at (0), and above (2) the $\alpha$ resonance. (b) The decay length $\tilde{d}_{x}^{\alpha}$ and wave length $\tilde{\lambda}_{x}^{\alpha}$, Eq. (21), as well as $\left|\tilde{k}_{x}^{\alpha}\right|^{-1}$, Eq. (19), plotted versus $\Delta^{\alpha}$. The three vertical dashed lines mark the values used in (a).

wave number $\bar{k}_{0}^{\alpha} \approx \frac{\pi}{L_{z}}$, for which Eq. (20) leads to the estimate $L_{x}^{\alpha} \approx \frac{1}{\pi \sqrt{\bar{\Gamma}^{\alpha}}} L_{z}$. For realistic values of the damping coefficient, $0.001<\bar{\Gamma}^{\alpha}<0.01$ [43], we obtain $10 L_{z}>L_{x}^{\alpha}>3 L_{z}$.

\section{THE AXIAL DEPENDENCY OF THE PRESSURE FOR A SEPARABLE ACTUATION}

Above, we derived analytical expressions for the characteristic length scales of the Green's function $g^{\alpha}(x)$ entering in Eq. (17c) for the pressure amplitude $P_{1}^{\alpha}(x)$. Now, we show analytically that the full 3D pressure at resonance separates into a product of the 2D resonance mode and a trivial axial dependency. We first calculate the axial variation of the pressure mode $p_{1}^{\alpha}$ for a given separable model-actuation $U_{1 \perp}$ having the dimensionless axial dependency $\psi_{\text {act }}(x)$,

$$
U_{1 \perp}(x, y, z)=\bar{U}_{1 \perp}(y, z) \psi_{\text {act }}(x) .
$$

In this case, the $3 \mathrm{D}$ mode $p_{1}^{\alpha}(x, y, z)$ in Eq. (17b) becomes a product of the $2 \mathrm{D}$ mode $\bar{p}_{1}^{\alpha}(y, z)$ and the dimensionless axial dependency $\chi^{\alpha}(x)$ of the pressure,

$$
\begin{aligned}
p_{1}^{\alpha}(x, y, z) & =\bar{p}_{1}^{\alpha}(y, z) \chi^{\alpha}(x), \\
\chi^{\alpha}(x) & =\left[\psi_{\text {act }} * g^{\alpha}\right](x),
\end{aligned}
$$

which by Eq. (17a) leads to the pressure $p_{1}(x, y, z)$,

$$
p_{1}(x, y, z)=\sum_{\alpha} \bar{p}_{1}^{\alpha}(y, z) \chi^{\alpha}(x), \quad \text { for any frequency } f .
$$

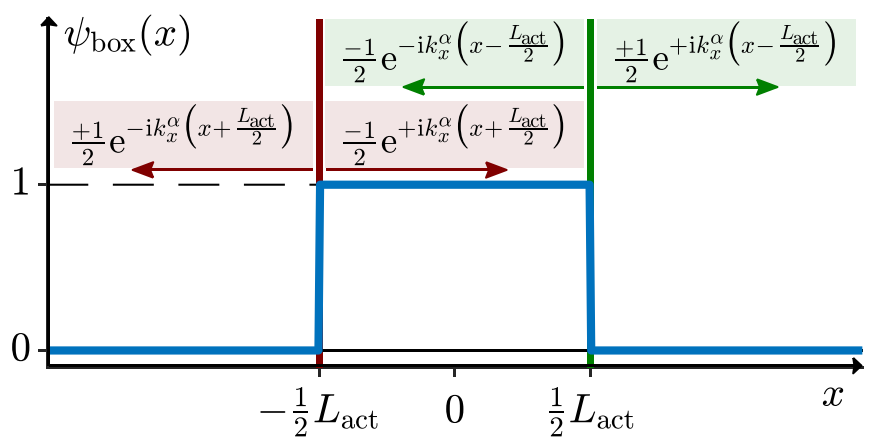

FIG. 4. The box-shaped axial dependency $\psi_{\text {act }}^{\text {box }}(x)$ (dark blue line) of the model actuation of width $L_{\text {act }}$, as well as the resulting traveling-wave components excited by the left step at $x=-\frac{1}{2} L_{\text {act }}$ (dark red arrows) and by the right step at $x=\frac{1}{2} L_{\text {act }}$ (light green arrows).

When actuating the system near one of the $2 \mathrm{D}$ resonances, say $\alpha=\alpha^{\prime}$, we obtain the simplified expression

$$
p_{1}(x, y, z) \approx \bar{p}_{1}^{\alpha^{\prime}}(y, z) \chi^{\alpha^{\prime}}(x), \text { for } f \approx \bar{f}^{\alpha^{\prime}} .
$$

\section{A. A box actuation with sharp steps}

In capillary-tube devices used for acoustic trapping, a piezoelectric transducer is usually placed below the capillary tube in a confined region [14-16,20,21,44]. To mimic such an actuation, we consider the box-shaped axial dependency $\psi_{\text {act }}^{\text {box }}(x)$ of the actuation, which is unity in the actuation region of length $L_{\text {act }}$ and sharply steps down to zero outside this region, as sketched in Fig. 4,

$$
\psi_{\text {act }}^{\text {box }}(x)= \begin{cases}1, & |x|<\frac{1}{2} L_{\text {act }}, \\ 0, & |x|>\frac{1}{2} L_{\text {act }} .\end{cases}
$$

Using this actuation in the convolution Eq. (24b) yields the axial dependency $\chi_{\text {box }}^{\alpha}(x)$ of the pressure,

$$
\chi_{\text {box }}^{\alpha}(x)= \begin{cases}1-e^{i k_{x}^{\alpha} \frac{L_{\text {act }}}{2}} \cos \left(k_{x}^{\alpha} x\right), & |x|<\frac{1}{2} L_{\text {act }}, \\ -i \sin \left(k_{x}^{\alpha} \frac{L_{\text {act }}}{2}\right) e^{i k_{x}^{\alpha}|x|}, & |x|>\frac{1}{2} L_{\text {act }} .\end{cases}
$$

By writing the sine and cosine factors in terms of traveling waves, it is found that two waves travel away from each step at $x= \pm \frac{1}{2} L_{\text {act }}$ as sketched in Fig. 4 .

In the limit $L_{\text {act }} \ll L_{x}^{\alpha}$, where the actuation is confined to a region much narrower than the axial pressure length scale $L_{x}^{\alpha}$ of Eq. (20), the axial dependency of the pressure is well approximated by a simplified expression $\chi_{\delta}^{\alpha}(x)$ as

$$
\chi_{\text {box }}^{\alpha}(x) \approx \chi_{\delta}^{\alpha}(x)=L_{\text {act }} g^{\alpha}(x), \quad \text { for } L_{\text {act }} \ll L_{x}^{\alpha},
$$

where $g^{\alpha}(x)$ is defined in Eq. (17d) and plotted in Fig. 3(a) for different frequencies.

\section{B. A box actuation with smooth steps}

In the following numerical validation, we consider the more realistic box-actuation function $U_{1 \perp}^{\text {num }}(x, y, z)=$ $\bar{U}_{1 \perp}^{\text {num }}(y, z) \psi_{\text {act }}^{\text {num }}(x)$, which separates as Eq. (23) with an axial dependency $\psi_{\mathrm{act}}^{\mathrm{num}}(x)$, similar to the box shape $\psi_{\mathrm{act}}^{\mathrm{box}}(x)$ in 
Eq. (27a), but which has smooth transistions of width $d_{\text {act }}$ at the steps $x= \pm \frac{1}{2} L_{\text {act }}$,

$$
\begin{aligned}
U_{1 \perp}^{\mathrm{num}}(x, y, z) & =\bar{U}_{1 \perp}^{\mathrm{num}}(y, z) \psi_{\mathrm{act}}^{\mathrm{num}}(x), \quad \text { at } \partial \Omega, \\
\psi_{\mathrm{act}}^{\mathrm{num}}(x) & =\frac{1}{1+e^{\frac{4\left(x-\frac{1}{2} L_{\text {act }}\right)}{d_{\text {act }}}}}-\frac{1}{1+e^{\frac{4\left(x+\frac{1}{2} L_{\text {act }}\right.}{d_{\text {act }}}}} .
\end{aligned}
$$

Here, $\bar{U}_{1 \perp}^{\text {num }}$ is defined in Eq. (13) and shown in Fig. 2.

\section{Numerical validation of the 3D mode method}

Using COMSOL Multiphysics as described in Sec. III, we validate the semianalytical Eq. (26) for $p_{1}(x, y, z)$ by the direct numerical solution $p_{1}^{\text {num }}$ of Eq. (4) in the capillary tube $\Omega$ sketched in Fig. 1, both actuated at the resonance frequency $f=\bar{f}^{1}$ of the fundamental mode $\alpha=1$ of the 2D cross section $\bar{\Omega}$. We assume that the tube is mirror-symmetric around the $y-z$ plane through $x=0$, and take the length of the computational domain to be $L_{x}^{\text {num }}=10 L_{x}^{1}=5.46 \mathrm{~cm}$, see Table II. At $x=0$ we impose the symmetry boundary condition $\partial_{x} p_{1}^{\text {num }}=0$, and at $x=L_{x}^{\text {num }}$ we place the perfectly matched layer (PML) mentioned in Sec. III to remove pressure wave reflections from the tube end. The wavy boundary $\partial \bar{\Omega}$ of the cross section $\bar{\Omega}$ is defined in Sec. III.

Using the 2D pressure mode $(9) \bar{p}_{1}^{1}(y, z)$ obtained from the eigenvalue problem (10), we construct the $3 \mathrm{D}$ pressure mode (24), as $p_{1}^{1}(x, y, z)=\bar{p}_{1}^{1}(y, z) \chi^{1}(x)$. In the following, we use the analytically known axial dependencies $\chi_{\text {box }}^{1}(x)$, Eq. (27b), and $\chi_{\delta}^{1}$, Eq. (18), of the pressure to estimate the pressure obtained numerically from the actuation profile $\psi_{\mathrm{act}}^{\mathrm{num}}(x)$ in Eq. (29b).

To quantify the numerical validation, we compute at the resonance $f=\bar{f}^{1}$ the relative deviation $\mathcal{E}\left(p_{1}^{1}, p_{1}^{\text {num }}\right)$ defined in Eq. (6) between the semianalytical 3D pressure mode $p_{1}^{1}$ with the box actuation $\psi_{\text {act }}^{\text {box }}(x)$ of Eq. (27a) and the direct numerical 3D pressure $p_{1}^{\text {num }}$ with the smoothen-box actuation $\psi_{\text {act }}^{\text {num }}(x)$ of Eq. (29b),

$$
\begin{aligned}
p_{1}^{1} & =\bar{p}_{1}^{1}(y, z) \chi_{\text {box }}^{1}(x), \quad \text { with } \psi_{\text {act }}^{\text {box }}(x) \text { at } \bar{f}^{1}, \\
p_{1}^{\text {num }} & =p_{1}(x, y, z), \quad \text { with } \psi_{\text {act }}^{\text {num }}(x) \text { at } \bar{f}^{1} .
\end{aligned}
$$

In Fig. 5, we study the axial dependency of the pressure for varying actuation step width $d_{\text {act }}$ and fixed actuation length of $L_{\text {act }}=2 L_{x}^{1}$, rescaled as in Eq. (22) by the characteristic length scale $L_{x}^{1}$,

$$
\tilde{d}_{\mathrm{act}}=\frac{d_{\mathrm{act}}}{L_{x}^{1}}, \quad \tilde{L}_{\mathrm{act}}=\frac{L_{\mathrm{act}}}{L_{x}^{1}} .
$$

In Fig. 5(b) is shown that for small $d_{\text {act }}$ the semianalytical expression (30a) is a good approximation for all $\tilde{x}$. For large $d_{\text {act }}$ it deviates significantly from the numerical solution (30b) inside the actuation region for $|\tilde{x}|<1$, whereas it remains a good approximation outside for $|\tilde{x}|<1$. This is quantified in the inset of Fig. 5(b), showing that the deviation $\mathcal{E}_{\text {box }}$ (solid line) is around $1 \%$ for a sufficiently narrow actuation step width $\tilde{d}_{\text {act }} \lesssim 0.4$.

In Fig. 6 we vary the actuation length $L_{\text {act }}$ and keep the actuation step width fixed at $\tilde{d}_{\text {act }}=0.1$. For all actuation lengths $\tilde{L}_{\text {act }}$, the semianalytical expression (30a) $\bar{p}_{1}^{1} \chi_{\text {box }}^{1}$ (dashed magenta lines) approximates well the full numerical
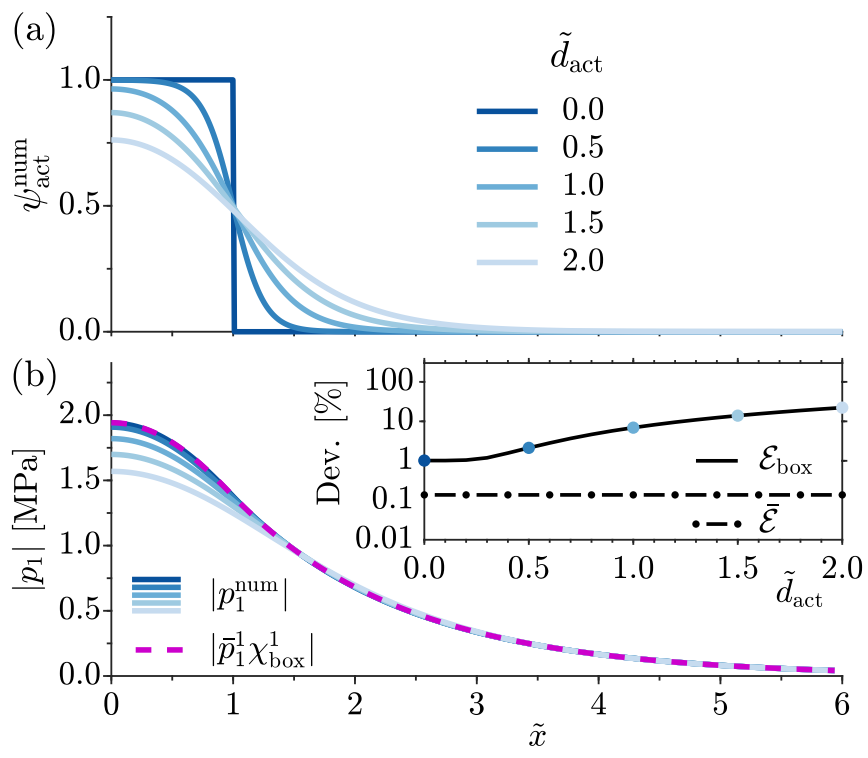

FIG. 5. The acoustic pressure for varying actuation step width $\tilde{d}_{\text {act }}$ and fixed actuation length $\tilde{L}_{\text {act }}=2$. (a) The actuation profile $\psi_{\text {act }}^{\text {num }}(x)$ used in the full numerical simulation for $\tilde{d}_{\text {act }}=0.0$ (dark blue) to $\tilde{d}_{\text {act }}=2.0$ (light blue). (b) Line plots of the magnitude of the acoustic pressure along the axis parallel to the $x$-axis shown in Fig. 2, with $\left|p_{1}^{1}\right|=\left|\bar{p}_{1}^{1} \chi_{\text {box }}^{1}\right|$ (magenta dashed line) from Eq. (30a), and $\left|p_{1}^{\text {num }}\right|$ (blue lines) from Eq. (30b) obtained from the 3D simulation by using the actuation of same color shown in (a). The inset shows the relative deviation (6) $\mathcal{E}_{\text {box }}$ (solid line) of the pressure mode $\bar{p}_{1}^{1}(y, z) \chi_{\text {box }}^{1}(x)$ from the numerical pressure $p_{1}^{\text {num }}$, as well as the deviation for the $2 \mathrm{D}$ calculation given in Sec. IV A, $\overline{\mathcal{E}}=0.14 \%$ (dot-dashed line).

solution $p_{1}^{\text {num }}(30 \mathrm{~b})$ (solid green lines), whereas $\bar{p}_{1}^{1}(y, z) \chi_{\delta}^{1}(x)$ (black dotted lines), see Eq. (18), as expected is only a good approximation in the narrow-actuation limit $\tilde{L}_{\text {act }} \ll 1$. In the inset of Fig. $6(\mathrm{~b})$ the relative deviations of these approximations from $p_{1}^{\text {num }}$ are quantified by $\mathcal{E}_{\text {box }}$ (solid line) and $\mathcal{E}_{\delta}$ (dotted line).

\section{TIME-AVERAGED ACOUSTIC QUANTITIES CLOSE TO RESONANCE}

In typical experiments on acoustofluidic devices, the $\mathrm{MHz}$ oscillation of the acoustic pressure $p_{1}$ is not observed directly. We therefore study the time-averaged acoustic quantities given in Eq. (5).

\section{A. Time-averaged quantities for a single mode}

We study a single-mode pressure resonance of the form $p_{1} \approx p_{1}^{\alpha}=\bar{p}_{1}^{\alpha}(y, z) \chi^{\alpha}(x)$, see Eq. (26). Inserting this form in Eq. (5) together with the rescaled axial coordinate $\tilde{x}=x / L_{x}^{\alpha}$ and the corresponding derivative $\partial_{\tilde{x}}=L_{x}^{\alpha} \partial_{x}$, both scaled with the characteristic axial length scale $L_{x}^{\alpha}$ from Eq. (20), we obtain the time-averaged quantities,

$$
\begin{aligned}
& E_{\mathrm{pot}}^{\alpha}=\bar{E}_{\mathrm{pot}}^{\alpha}\left|\chi^{\alpha}\right|^{2}, \\
& E_{\text {kin }}^{\alpha}=\bar{E}_{\text {kin }}^{\alpha}\left|\chi^{\alpha}\right|^{2}+\frac{\bar{E}_{\mathrm{pot}}^{\alpha}}{\left(k_{0} L_{x}^{\alpha}\right)^{2}}\left|\partial_{\tilde{x}} \chi^{\alpha}\right|^{2} \approx \bar{E}_{\mathrm{kin}}^{\alpha}\left|\chi^{\alpha}\right|^{2},
\end{aligned}
$$



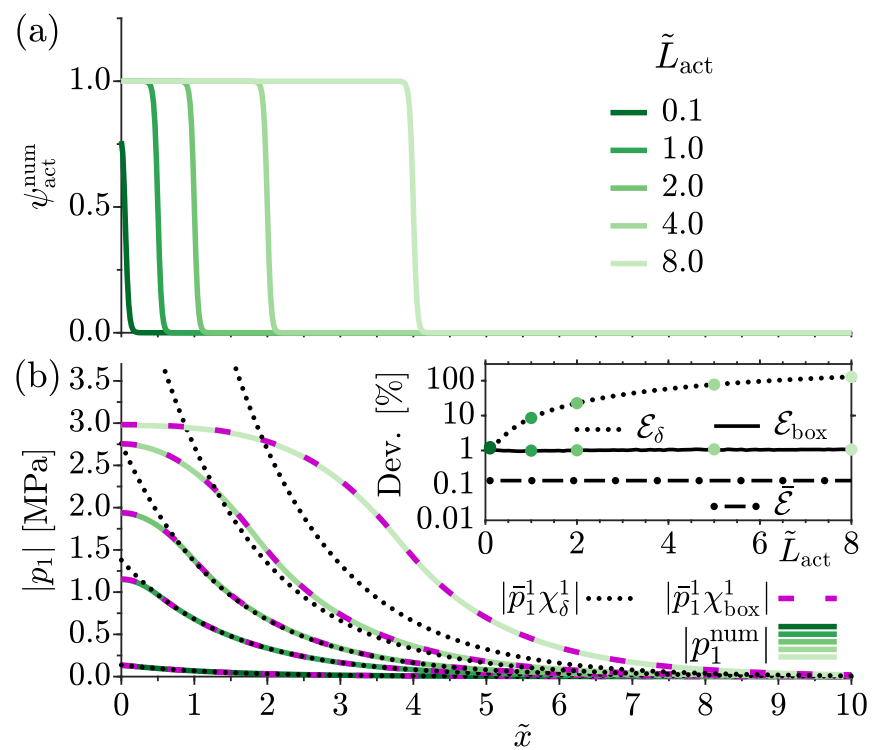

FIG. 6. The acoustic pressure for varying actuation length $\tilde{L}_{\text {act }}$ and fixed actuation step width $\tilde{d}_{\text {act }}=0.1$. (a) The actuation profile $\psi_{\text {act }}^{\text {num }}(x)$ used in the full numerical simulations for $\tilde{L}_{\text {act }}=0.1$ (dark green) to $\tilde{L}_{\text {act }}=8.0$ (light green). (b) Line plots of the magnitude of the acoustic pressure along the axis parallel to the $x$ axis shown in Fig. 2. The green graphs show the pressure $p_{1}^{\text {num }}$ obtained from the $3 \mathrm{D}$ simulation by using the actuation of same color shown in (a). The dashed magenta lines show $p_{1}^{1}=\bar{p}_{1}^{1} \chi_{\text {box }}^{1}$ from Eq. (30b), and the dotted black lines show the pressure $\bar{p}_{1}^{1} \chi_{\delta}^{1}$ valid in limit $\tilde{L}_{\text {act }} \ll 1$, see Eq. (18). The inset shows the deviation $\mathcal{E}$ from to the reference pressure $p_{1}^{\text {num }}$ Eq. (6), for $\bar{p}_{1}^{1} \chi_{\text {box }}^{1}\left(\mathcal{E}_{\text {box }}\right.$, solid), for $\bar{p}_{1}^{1} \chi_{\delta}^{1}\left(\mathcal{E}_{\delta}\right.$, dotted $)$, as well as the deviation for the $2 \mathrm{D}$ calculation given in Sec. IV A, $\overline{\mathcal{E}}=0.14 \%$ (dot-dashed line).

$$
\begin{aligned}
E_{\mathrm{ac}}^{\alpha} & \approx \bar{E}_{\mathrm{ac}}^{\alpha}\left|\chi^{\alpha}\right|^{2}, \\
U_{\mathrm{rad}}^{\alpha} & \approx \bar{U}_{\mathrm{rad}}^{\alpha}\left|\chi^{\alpha}\right|^{2}, \\
\boldsymbol{F}_{\mathrm{rad}}^{\alpha} & \approx \overline{\boldsymbol{F}}_{\mathrm{rad}}^{\alpha}\left|\chi^{\alpha}\right|^{2}-\sqrt{\bar{\Gamma}^{\alpha}} \bar{k}_{0}^{\alpha} \bar{U}_{\mathrm{rad}}^{\alpha} \partial_{\tilde{x}}\left|\chi^{\alpha}\right|^{2} \boldsymbol{e}_{x}, \\
\boldsymbol{S}_{\mathrm{ac}}^{\alpha} & =\overline{\boldsymbol{S}}_{\mathrm{ac}}^{\alpha}\left|\chi^{\alpha}\right|^{2}+\sqrt{\bar{\Gamma}^{\alpha}} c_{\mathrm{ff}} \bar{E}_{\mathrm{pot}}^{\alpha} \operatorname{Im}\left[2\left(\chi^{\alpha}\right)^{*} \partial_{\tilde{x}} \chi^{\alpha}\right] \boldsymbol{e}_{x} .
\end{aligned}
$$

Here, the overbar denote a cross-section quantity obtained by using the cross-section resonance pressure $\bar{p}_{1}^{\alpha}(y, z)$ in Eq. (5). In Eqs. (32b)-(32e) we used that $\left(k_{0} L_{x}^{\alpha}\right)^{-2} \approx \bar{\Gamma}^{\alpha}$, see Eq. (20), which is here assumed to be much smaller than unity. We note that the time-averaged quantities listed in Eq. (32) have three different axial dependencies: $\left|\chi^{\alpha}\right|^{2}$ which is the axial dependency of $E_{\mathrm{kin}}^{\alpha}, E_{\mathrm{pot}}^{\alpha}, E_{\mathrm{ac}}^{\alpha}, U_{\mathrm{rad}}^{\alpha},\left(\boldsymbol{F}_{\mathrm{rad}}^{\alpha}\right)_{y z}$, and $\left(\boldsymbol{S}_{\mathrm{ac}}^{\alpha}\right)_{y z}$. $\partial_{\tilde{x}}\left|\chi^{\alpha}\right|^{2}$ which is the axial dependency of the axial radiation force $F_{\text {rad }, x}^{\alpha}$. And finally $\operatorname{Im}\left[2\left(\chi^{\alpha}\right)^{*} \partial_{\tilde{x}} \chi^{\alpha}\right]$ which is the axial dependency of the axial energy flux density $S_{\mathrm{ac}, x}^{\alpha}$.

\section{B. Time-averaged quantities for the box actuation}

In Fig. 7, we use the box actuation with $\chi^{\alpha}=\chi_{\text {box }}^{\alpha}$, see Eq. (27b), to make contour plots in the $\tilde{L}_{\text {act }}-\tilde{x}$ plane of the axial dependency of the time-averaged quantities listed in Eq. (32). For each quantity, we choose the rescaled frequency $\Delta^{\alpha} \approx \frac{f_{0}-f_{0}^{\alpha}}{\frac{1}{2} f_{0} \bar{\Gamma}^{\alpha}}$, see Eq. (19), to obtain the largest possible value
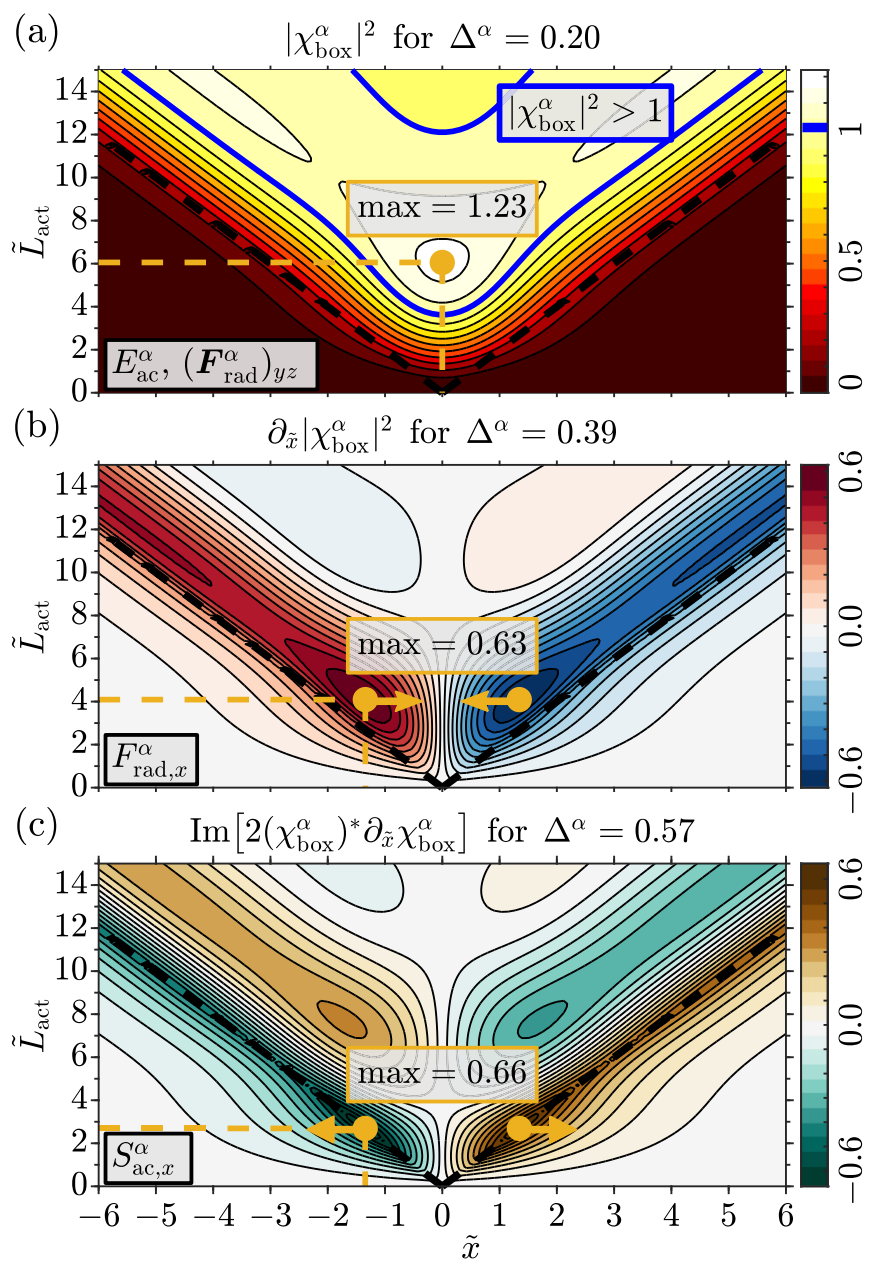

FIG. 7. The axial dependency of the time-averaged acoustic quantities of Eq. (32) obtained by using $\chi_{\text {box }}^{\alpha}$ from Eq. (27b) as the axial dependency of the pressure. In each plot, the rescaled frequency $\Delta^{\alpha} \approx \frac{f_{0}-f_{0}^{\alpha}}{\frac{1}{2} f_{0} \bar{\Gamma}^{\alpha}}$, Eq. (19), is chosen to maximize the corresponding physical quantity, and the maximum value is marked by yellow points. The black dashed lines show the actuation edge $\tilde{x}= \pm \frac{1}{2} \tilde{L}_{\text {act }}$. (a) The axial dependency $\left|\chi_{\mathrm{box}}^{\alpha}\right|^{2}$ of the acoustic energy density $E_{\mathrm{ac}}^{\alpha}$, and the cross-sectional radiation force $\left(\boldsymbol{F}_{\text {rad }}\right)_{y z}$, where the contours are separated by 0.1 and the thick blue lines mark the area where the axial dependency of $\left|\chi_{\text {box }}^{\alpha}\right|^{2}$ exceeds unity. (b) The axial dependency $\partial_{\tilde{x}}\left|\chi_{\text {box }}^{\alpha}\right|^{2}$ of the axial radiation force $F_{\text {rad }, x}^{\alpha}$. (c) The axial dependency $\operatorname{Im}\left[2\left(\chi_{\mathrm{box}}^{\alpha}\right)^{*} \partial_{\tilde{x}} \chi_{\mathrm{box}}^{\alpha}\right]$ of the axial energy flux density $S_{\mathrm{ac}, x}^{\alpha}$.

of that quantity, i.e. to optimize $\left|\overline{\mathcal{G}}^{\alpha}\right|^{2}\left|\chi_{\text {box }}^{\alpha}\right|^{2},\left|\overline{\mathcal{G}}^{\alpha}\right|^{2} \partial_{\tilde{x}}\left|\chi_{\text {box }}^{\alpha}\right|^{2}$, and $\left|\overline{\mathcal{G}}^{\alpha}\right|^{2} \operatorname{Im}\left[2\left(\chi_{\text {box }}^{\alpha}\right)^{*} \partial_{\tilde{x}} \chi_{\text {box }}^{\alpha}\right]$, respectively.

In Fig. 7(a) is shown a contour plot of the axial dependency $\left|\chi_{\text {box }}^{\alpha}(x)\right|^{2}$ of the acoustic energy density $E_{\mathrm{ac}}^{\alpha}$, Eq. (32c), and cross-sectional radiation force $\left(\boldsymbol{F}_{\text {rad }}^{\alpha}\right)_{y, z}, \mathrm{Eq}$. (32e). The blue contour delimit the region where the 3D acoustic energy density $E_{\mathrm{ac}}^{\alpha}$ is larger than the $2 \mathrm{D}$ acoustic energy density $\bar{E}_{\mathrm{ac}}^{\alpha}$. The orange dot marks the maximum obtainable acoustic energy density which is $\max \left\{E_{\text {ac }}^{\alpha}\right\}=1.23 \bar{E}_{\text {ac }}^{\alpha}$, found at the optimal actuation length $\tilde{L}_{\text {act }} \approx 6.1$.

In Fig. 7(b) is shown a contour plot of the axial dependency $\partial_{\tilde{x}}\left|\chi_{\text {box }}^{\alpha}(x)\right|^{2}$ of the axial acoustic radiation force $F_{\text {rad }, x}^{\alpha}$, see Eq. (32e). The orange dots mark the maximum obtainable axial trapping force which is found to be $\max \left\{F_{\text {rad }, x}^{\alpha}\right\}=$ 
$\mp 0.63 \sqrt{\bar{\Gamma}^{\alpha}} \bar{k}_{0}^{\alpha} \bar{U}_{\text {rad }}^{\alpha}$, for the optimal actuation length $\tilde{L}_{\text {act }} \approx 4.1$. For this optimal value, the force is largest at the axial position $\tilde{x}= \pm 1.3$, which is around $17 \%$ inside the actuation region.

Finally, in Fig. 7(c) is shown a contour plot of the axial dependency $\operatorname{Im}\left[2\left(\chi_{\text {box }}^{\alpha}\right)^{*} \partial_{\tilde{x}} \chi_{\text {box }}^{\alpha}\right]$ of the axial acoustic energy flux density $S_{\mathrm{ac}, x}^{\alpha}$; see Eq. (32f). Clearly, the energy is always transported away from the actuation region at the edges of the actuation domain $\tilde{x} \approx \pm \frac{1}{2} \tilde{L}_{\text {act }}$. The orange dots mark the largest obtainable axial energy flux density which is $\max \left\{S_{\mathrm{ac}, x}^{\alpha}\right\}= \pm 0.66 \sqrt{\bar{\Gamma}^{\alpha}} c_{\mathrm{ff}} \bar{E}_{\mathrm{pot}}^{\alpha}$.

\section{Example: A standing half-wave resonance in a rectangular cross section}

A standard device for acoustic trapping is the capillary tube with the rectangular cross section $0<y<L_{y}$ and $0<$ $z<L_{z}$, where a standing-half-wave resonance in the vertical $z$ direction is excited [14-16,20,21,44]. Using Eq. (7) with $\bar{k}_{0}^{01}=\frac{\pi}{L_{z}}$ for the 2D pressure mode $\bar{p}_{1}^{01}=\bar{P}_{1}^{01} \overline{\mathcal{G}}^{01} \cos \left(\bar{k}_{0}^{01} z\right)$, we evaluate the cross-sectional radiation force $F_{\mathrm{rad}, z}^{01}(x, z)$ from Eq. (32e) as

$$
\frac{F_{\mathrm{rad}, z}^{01}(x, z)}{4 \pi a^{3} \bar{k}_{0}^{01}\left\langle\bar{E}_{\mathrm{ac}}^{01}\right\rangle} \approx \Phi \sin \left(2 \bar{k}_{0}^{01} z\right)\left|\chi^{01}(x)\right|^{2},
$$

where $\Phi=\frac{1}{3} f_{0}+\frac{1}{2} f_{1}$ is the usual acoustic contrast factor $[23,26,27]$, and $\left\langle\bar{E}_{\text {ac }}^{01}\right\rangle=\frac{1}{4} \kappa_{\mathrm{ff}}\left|\bar{P}^{01} \overline{\mathcal{G}}^{01}\right|^{2}$ is the spatial average of the acoustic energy density Eq. (32c) in the cross section $\bar{\Omega}$. Similarly, we use Eqs. (7) and (32e) to evaluate the axial radiation force,

$$
\frac{F_{\mathrm{rad}, x}^{01}(x, z)}{4 \pi a^{3} \bar{k}_{0}^{01}\left\langle\bar{E}_{\mathrm{ac}}^{01}\right\rangle} \approx\left[\frac{1}{2} f_{1}-\Phi \cos ^{2}\left(\bar{k}_{0}^{01} z\right)\right] \sqrt{\bar{\Gamma}^{01}} \partial_{\tilde{\chi}}\left|\chi^{01}\right|^{2} .
$$

From Eqs. (33a) and (33b), we calculate the ratio between the maximum axial radiation force and the maximum cross section radiation force for the single standing-wave resonance by using $\max \left\{\partial_{\tilde{x}}\left|\chi^{01}(x)\right|^{2}\right\} \sim 2 \max \left\{\left|\chi^{01}(x)\right|^{2}\right\}$,

$$
\begin{aligned}
\frac{\max \left\{F_{\mathrm{rad}, x}^{01}\right\}}{\max \left\{F_{\mathrm{rad}, z}^{01}\right\}} & \approx \frac{f_{1}}{\Phi} \sqrt{\bar{\Gamma}^{01}}=\frac{2}{1+\frac{2 f_{0}}{3 f_{1}}} \sqrt{\bar{\Gamma}^{01}}, \\
& \approx 0.21 \sqrt{\bar{\Gamma}^{01}}, \text { for polystyrene particles. }
\end{aligned}
$$

In the last step we use the scattering coefficients $f_{0}=0.443$ and $f_{1}=0.034$ for large polystyrene particles [26]. As examples, Ley and Bruus [36] studied numerically the pyrex-glass capillary tubes named " $\mathrm{C} 1$ " (used by Hammerström et al. [20] with inner dimensions $L_{y}=2 \mathrm{~mm}$ and $L_{z}=0.2 \mathrm{~mm}$ ) and "C5" (proposed by the authors with inner dimensions $L_{y}=0.5 \mathrm{~mm}$ and $L_{z}=0.2 \mathrm{~mm}$ ). In both cases, they found the quality factor for the standing half-wave resonance in the $z$ direction to be $Q=53$, corresponding to the damping coefficient $\bar{\Gamma}^{01}=\frac{1}{53}$. Using this value in (33c) gives the ratio $\frac{1}{35}$, where Ley and Bruus found the ratio to be $\frac{0.44 \mathrm{pN}}{22 \mathrm{pN}}=\frac{1}{50}$ for $\mathrm{C} 1$ (see their Fig. 6) and $\frac{0.13 \mathrm{pN}}{7 \mathrm{pN}}=\frac{1}{54}$ for $\mathrm{C} 5$ (see their Fig. 9). Hence, even though Eq. (33c) is obtained from a hard-wall analysis, it predicts values close to the full simulation where the surrounding glass capillary is included.
Furthermore, for the capillary tube $\mathrm{C} 1$ with the experimentally found resonance frequency $f=\frac{1}{2 \pi} k_{0} c_{\mathrm{fl}}=3.970 \mathrm{MHz}$ and transducer length $L_{\text {act }}=1160 \mu \mathrm{mm}$ [20], we calculate $\tilde{L}_{\text {act }}=L_{\text {act }} k_{0} \sqrt{\bar{\Gamma}^{01}}=2.6$. From Figs. 7(a) and 7(b) follows the prediction that the acoustic energy density $E_{\mathrm{ac}}^{\alpha}$ and trapping force $F_{\text {rad }, x}^{\alpha}$ may be approximately doubled by doubling $L_{\text {act }}$.

\section{DISCUSSION}

The theory presented in Sec. IV relies on the main assumption that the 2D resonance modes in an arbitrary cross section can be written as in Eq. (9) for wave numbers $k_{0}$ very close to the cross-section eigenvalues $\bar{k}_{0}^{\alpha}$. Whereas this generalization is not proven mathematically, it is physically reasonable, and we have validated it numerically in Fig. 2 with a relative deviation of $0.14 \%$. We note that the eigenfunctions $\bar{R}^{\alpha}(y, z)$ in the cross section do not exactly form a complete set, because the eigenvalues of the eigenvalue problem Eq. (10) has a small imaginary part. However, in the limit $k_{0} \delta_{\mathrm{s}} \ll 1$ the viscous boundary layer introduces a minute imaginary part to the eigenvalues, and thus Eq. (9) is a good approximation. It may be possible to come up with a special cross section where our theory fails, but it does apply to all capillary tube cross sections used in the experiments that are reported in the literature.

We have presented detailed results for the special simplifying, but experimentally relevant, condition that the actuation frequency is near a resonance characterized by a single mode that does not overlap with other modes, such that the 3D pressure $p_{1}$ is described by only a single term of the sum Eq. (17). We emphasize, however, that this is not a necessary condition, as the general theory allows both for nonresonant actuation and for multiple overlapping modes. In fact, we have done equally successful validations for frequencies away from resonance, where more modes are taken into account.

We have considered the actuation to have a box-shaped axial dependency given by Eq. (27a) to mimic a piezoelectric transducer confined in the axial direction to a length $L_{\text {act }}$. In a realistic glass-capillary system, the motion of the wall will be more complicated as found from the numerical simulations by Ley and Bruus [36] and the simulations and experiments by Reichert et al. [45]. Nevertheless, when calculating the ratio between the axial and cross-sectional radiation force in the end of Sec. VIC and using the damping coefficient $\bar{\Gamma}^{1}=\frac{1}{53}$ found from the numerical simulation by Ley and Bruus, we almost reproduce their values, namely, $\frac{F_{\text {rad }, x}}{F_{\text {rad. }, z}} \approx \frac{1}{50}$. This agreement indicates that the predictions from our theory of the axial variations of the pressure remain valid for more complicated wall actuation, and that the important effect from the capillary walls is well described by a change in the damping coefficient $\bar{\Gamma}^{\alpha}$ for the mode. The probable cause for this increased damping factor of the fluid resonance is not dissipation in the capillary tube but instead an axial transport of energy in the solid away from the fluid, as pointed out by Ley and Bruus [36]. This can be seen from Eq. (32f), which states that the axial transport $\left(\boldsymbol{S}_{\mathrm{ac}}\right)_{x}$ of energy is proportional to the speed of sound $c_{\mathrm{ff}}$, and because the speed of sound in the capillary tube is usually larger than in the fluid, energy 
is efficiently transported away from the trapping region. For example, the longitudinal sound speed in pyrex glass is 3.7 times larger than in water [46].

\section{CONCLUSION AND OUTLOOK}

We have presented a semianalytical method to calculate the acoustic pressure in a long, straight capillary tube of arbitrary cross section with a localized ultrasound actuation at the walls. Moreover, we have analytically derived the axial dependencies Eq. (32) of the time-averaged response and used it to derive an expression for the key aspect in the acoustic trap, namely, the axial acoustic radiation force Eq. (33) acting on suspended particles. The viscous boundary layer is taken into account through an effective boundary condition Eq. (4b), which is valid when the width $\delta_{\mathrm{s}}$ of the viscous boundary layer is much smaller than both the acoustic wavelength $\left(k_{0} \delta_{\mathrm{s}} \ll 1\right)$ and the radius of curvature of the cross section. This condition is usually satisfied in typical experiments.

In Eq. (9), the acoustic 2D cross-section resonance mode $\bar{p}_{1}^{\alpha}(y, z)$ in an arbitrary cross section was obtained by a generalization of the well-studied case Eq. (7) of a rectangular cross section. The $2 \mathrm{D}$ mode $\bar{p}_{1}^{\alpha}(y, z)$ can be found analytically for integrable shapes, such as rectangles, circles and ellipses, and otherwise numerically as shown in Fig. 1 . The theory results in the correct amplitude and phase of $\bar{p}_{1}^{\alpha}(y, z)$ by combining the eigenvalue $\bar{k}_{\mathrm{c}}^{\alpha}$ and the dimensionless eigenfunction $\bar{R}^{\alpha}$ of Eq. (10) with the actuation function $\bar{U}_{1 \perp}$ of Eq. (4d) and the inclusion of the viscous boundary layer through the boundary operator $\mathcal{D}_{\perp}$ in Eq. (4c).

From the $2 \mathrm{D}$ pressure modes $\bar{p}_{1}^{\alpha}(y, z)$ in the cross section, known for frequencies near resonance, we derived in Eq. (17b) the 3D pressure modes $p_{1}^{\alpha}(x, y, z)$. The sum over $\alpha$ of these modes, see Eq. (17a), constitutes the full 3D pressure $p_{1}(x, y, z)$ including the axial dependency and valid for all frequencies. In Eq. (20) we extracted for 3D resonance mode $p_{1}^{\alpha}$ the characteristic axial length scale $L_{x}^{\alpha}=\left(\bar{k}_{0}^{\alpha} \sqrt{\bar{\Gamma}^{\alpha}}\right)^{-1}$, where $\bar{k}_{0}^{\alpha}$ is the $2 \mathrm{D}$ resonance wave number and $\bar{\Gamma}^{\alpha}$ is the 2D damping coefficient. Because the axial radiation force $\left(\boldsymbol{F}_{\mathrm{rad}}^{\alpha}\right)_{x}$ and the axial energy flux density $\left(\boldsymbol{S}_{\mathrm{ac}}^{\alpha}\right)_{x}$ of resonance mode $\alpha$, depend on the axial gradient of the pressure, we find in Eqs. (32e) and (32f) that these are both proportional to $\sqrt{\bar{\Gamma}^{\alpha}}$. These analytical results constitute the main theoretical insight obtained by our mode analysis. Furthermore, from a purely numerical-modeling point of view, the theoretical method implies a drastic reduction in the computer-memory requirements and in computation times, because the full 3D system can be obtained from a 2D simulation of the crosssection eigenproblem combined with the analytical expressions for the axial dependencies. This reduction will facilitate any numerical parametric study of the 3D acoustic trap.

To further study the physics of the acoustic trap, we chose a box-shaped actuation which mimics a piezoelectric transducer attached to the capillary walls in a confined region of length $L_{\text {act }}$, and which allows for analytic solutions. In Fig. 7 is shown the resulting axial dependencies of the acoustic energy density $E_{\mathrm{ac}}^{\alpha}$, the cross-sectional acoustic radiation force $\left(\boldsymbol{F}_{\text {rad }}\right)_{y z}$, the axial acoustic radiation force $F_{\text {rad }, x}^{\alpha}$, and the axial energy flux density $S_{\text {rad }, x}^{\alpha}$. Remarkably, we found an optimal actuation length $L_{\text {act }} \approx 2-5 L_{x}^{\alpha}$ that maximizes these quantities. Furthermore, whereas the maximum acoustic energy density $E_{\mathrm{ac}}^{\alpha}$ is found in the center of the channel, maximum axial radiation force $F_{\text {rad }, x}^{\alpha}$ is located around $17 \%$ inside the actuation region, and the maximum axial energy flux density $S_{\text {rad }, x}^{\alpha}$ is located at the edge of the actuation region.

We validated numerically our theory in Figs. 5 and 6 for the 3D system shown in Fig. 1 near the resonance $\alpha=1$, by using the box-shaped actuation given in Eq. (27a). We found a relative agreement around $1 \%$ between theory and simulation, even when the box-shaped actuation had smooth steps. This agreement is satisfactory as the theory was developed in the limit of a small boundary-layer width $\left(k_{0} \delta_{\mathrm{s}} \ll 1\right)$, and $k_{0} \delta_{\mathrm{s}}=$ 0.0024 in the numerical model.

The presented theory motivates further studies of capillary tubes. One obvious extension of this work is to compute the acoustic streaming, in particular the horizontal in-plane streaming rolls observed by Hammarström, Laurell, and Nilsson [20] and by Lei, Glynne-Jones, and Hill [15]. The streaming can be computed numerically by combining the presented theory with the recently published methods of calculating the time-averaged streaming velocity [30,37]. Another future study, would be to include the elastic walls in the mode analysis. For example, by combining the wall velocity $U_{1 \perp}$ obtained from a full 3D numerical simulation with the solution of the 2D eigenvalue problem Eq. (10), the coupling strength $P_{1}^{\alpha}$ for each pressure mode $p_{1}^{\alpha}$ can be calculated from Eq. (17). In this way the relative importance of each pressure mode in the acoustic trap can be characterized. A last example of further work is to investigate the loss of acoustic energy in the fluid into the solid as briefly discussed in the last paragraph of Sec. VII.

We have provided theoretical predictions of the axial variation of the acoustic fields in capillary tubes and pointed out that there is an optimal actuation length leading to a maximum acoustic radiation force, both in the axial and cross-sectional directions. Our analysis provides a theoretical understanding of the complicated 3D characteristics of acoustofluidics in capillary tubes, and in long, straight channels in general. Our resulting expressions can be used to aid in the design of acoustic trapping devices, and we hope that our work will inspire further systematic experimental characterization and optimization of acoustic traps.

\section{APPENDIX A: THE FOURIER TRANSFORM AND THE CONVOLUTION RELATIONS}

We define the Fourier transform $\mathcal{F}_{x}$ and the inverse Fourier transform $\mathcal{F}_{k}^{-1}$ as

$$
\begin{aligned}
& \hat{\phi}(k)=\mathcal{F}_{x}[\phi(x)](k)=\int_{-\infty}^{\infty} \phi(x) e^{-i k x} d x, \\
& \phi(x)=\mathcal{F}_{k}^{-1}[\hat{\phi}(k)](x)=\int_{-\infty}^{\infty} \hat{\phi}(k) e^{+i k x} \frac{d k}{2 \pi} .
\end{aligned}
$$

For this convention, the convolution relations are

$$
\begin{aligned}
\mathcal{F}\left[\phi_{1} * \phi_{2}\right] & =\mathcal{F}\left[\phi_{1}\right] \mathcal{F}\left[\phi_{2}\right], \\
\mathcal{F}\left[\phi_{1} \phi_{2}\right] & =\mathcal{F}\left[\phi_{1}\right] * \mathcal{F}\left[\phi_{2}\right],
\end{aligned}
$$


where the in-line asterisk denote the convolution,

$$
[f * g](x)=\int_{-\infty}^{\infty} f\left(x^{\prime}\right) g\left(x-x^{\prime}\right) d x^{\prime} .
$$

\section{APPENDIX B: DETAILS ABOUT THE USE OF THE BOUNDARY OPERATOR $\mathcal{D}_{\perp}$ IN Eq. (15)}

The boundary operator $\mathcal{D}_{\perp}$ defined in Eq. (4c) takes into account the viscous boundary layer. To be able to compare Eq. (15) for $\hat{p}_{1}$ to Eq. (4) for $\bar{p}_{1}$, we need to express $\mathcal{D}_{\perp}$ in terms of $k_{\mathrm{c}}^{2}-k_{x}^{2}$. This is achieved by subtracting and adding $k_{x}^{2}$ as $\mathcal{D}_{\perp}=\partial_{\perp}+\frac{i}{k_{\mathrm{s}}}\left[\left(k_{\mathrm{c}}^{2}-k_{x}^{2}\right)+k_{x}^{2}+\partial_{\perp}^{2}\right]$. Close to the poles we have $k_{x} \approx k_{x}^{\alpha}$, and thus Eq. (16b) gives $k_{\mathrm{c}}^{2}-k_{x}^{2} \approx\left(\bar{k}_{\mathrm{c}}^{\alpha}\right)^{2}$, whereby $\mathcal{D}_{\perp} \approx \partial_{\perp}+\frac{i}{k_{\mathrm{s}}}\left[\left(\bar{k}_{\mathrm{c}}^{\alpha}\right)^{2}+\left(k_{x}^{\alpha}\right)^{2}+\partial_{\perp}^{2}\right]$. If we assume $\left(\bar{k}_{\mathrm{c}}^{\alpha}\right)^{2} \gg\left(k_{x}^{\alpha}\right)^{2}$, then we may ignore $\left(k_{x}^{\alpha}\right)^{2}$. Later, this assumption is proven correct by noticing that Eq. (19) yields $\left(k_{x}^{\alpha}\right)^{2} \sim$ $\bar{\Gamma}^{\alpha}\left(\bar{k}_{\mathrm{c}}^{\alpha}\right)^{2}$ with $\bar{\Gamma}^{\alpha} \ll 1$. Consequently, Eq. (16) for the Fourier transform $\hat{p}_{1}$ at $k_{x}$-values close to the complex poles $k_{x}^{\alpha}$ is valid to $\mathcal{O}\left(\bar{\Gamma}^{\alpha}\right)$.

\section{APPENDIX C: DETAILS IN APPLYING THE RESIDUE THEOREM}

To obtain the residues $\operatorname{Res}\left(\hat{p}_{1}\left(k_{x} ; y, z\right) e^{i k_{x} x}, k_{x}^{\alpha}\right)$ used in Eq. (14b), we first rewrite Eq. (16a) by inserting $\hat{U}_{1 \perp}\left(k_{x} ; y, z\right)=\int_{-\infty}^{\infty} U_{1 \perp}\left(x^{\prime}, y, z\right) e^{-i k_{x} x^{\prime}} d x^{\prime}$,

$$
\begin{aligned}
& \hat{p}_{1}\left(k_{x} ; y, z\right) e^{i k_{x} x} \\
& \quad \approx \frac{-\left(\bar{k}_{\mathrm{c}}^{\alpha}\right)^{2} \bar{R}^{\alpha}}{\left(k_{x}\right)^{2}-\left(k_{x}^{\alpha}\right)^{2}} \frac{\int_{\partial \bar{\Omega}} \int_{-\infty}^{\infty} U_{1 \perp}\left(x^{\prime}, y, z\right) e^{i k_{x}\left(x-x^{\prime}\right)} d x^{\prime} \bar{R}^{\alpha} d l}{\int_{\bar{\Omega}}\left(\bar{R}^{\alpha}\right)^{2} d A} .
\end{aligned}
$$

This expression is valid for $k_{x}$ close to the simple poles given by $\pm k_{x}^{\alpha}= \pm \sqrt{k_{\mathrm{c}}^{2}-\left(\bar{k}_{\mathrm{c}}^{\alpha}\right)^{2}}$; see Eq. (16b). Based on Eq. (C1), the integral in Eq. (14a) is calculated using the residue theorem over a closed contour $\gamma$ in the complex $k_{x}$-plane chosen as follows: (1) For $x-x^{\prime}>0$, the integrand Eq. (C1) vanishes for $k_{x} \rightarrow i \infty$, and we choose the closed contour $\gamma$ to be the counterclockwise contour consisting of the real $\operatorname{Re}\left(k_{x}\right)$ axis connected to a semicircle of radius $\left|k_{x}\right| \rightarrow \infty$ in the upper complex $k_{x}$ plane. This contour encloses the residues at $k_{x}=$ $+k_{x}^{\alpha}=+\sqrt{k_{\mathrm{c}}^{2}-\left(\bar{k}_{\mathrm{c}}^{\alpha}\right)^{2}}$ having positive imaginary part. (2) For $x-x^{\prime}<0$, the integrand Eq. (C1) vanishes for $k_{x} \rightarrow-i \infty$, and we choose the closed contour $\gamma$ to be the clock-wise contour consisting of the real $\operatorname{Re}\left(k_{x}\right)$-axis connected to a semicircle of radius $\left|k_{x}\right| \rightarrow \infty$ in the lower complex $k_{x}$-plane. This contour encloses the residues at $k_{x}=-k_{x}^{\alpha}=-\sqrt{k_{\mathrm{c}}^{2}-\left(\bar{k}_{\mathrm{c}}^{\alpha}\right)^{2}}$ having negative imaginary part. In either case (1) or (2), the residues inside the closed contour $\gamma$ are

$$
\begin{aligned}
& \operatorname{Res}\left(\hat{p}_{1}\left(k_{x} ; y, z\right) e^{i k_{x} x}, k_{x}^{\alpha}\right) \\
& =\frac{-\left(\bar{k}_{\mathrm{c}}^{\alpha}\right)^{2} \bar{R}^{\alpha}}{2 k_{x}^{\alpha}} \frac{\int_{\partial \bar{\Omega}} \int_{-\infty}^{\infty} U_{1 \perp}\left(x^{\prime}, y, z\right) e^{i k_{x}^{\alpha}\left|x-x^{\prime}\right|} d x^{\prime} \bar{R}^{\alpha} d l}{\int_{\bar{\Omega}}\left(\bar{R}^{\alpha}\right)^{2} d A} \\
& =-i \overline{\mathcal{G}}^{\alpha}\left(k_{\mathrm{c}}\right) \bar{R}^{\alpha} \frac{\int_{\partial \bar{\Omega}} \int_{-\infty}^{\infty} U_{1 \perp}\left(x^{\prime}, y, z\right) g\left(x-x^{\prime}\right) d x^{\prime} \bar{R}^{\alpha} d l}{\int_{\bar{\Omega}}\left(\bar{R}^{\alpha}\right)^{2} d A},
\end{aligned}
$$

where we inserted $\overline{\mathcal{G}}^{\alpha}\left(k_{\mathrm{c}}\right)$ from Eq. (9c) and introduced the normalized function $g^{\alpha}(x)$ given by Eq. (17d). Finally, using the residues Eq. (C2) in the sum Eq. (14b), we obtain Eq. (17) for the acoustic pressure $p_{1}$.
[1] T. Laurell, F. Petersson, and A. Nilsson, Chip integrated strategies for acoustic separation and manipulation of cells and particles, Chem. Soc. Rev. 36, 492 (2007).

[2] M. K. Tan, R. Tjeung, H. Ervin, L. Y. Yeo, and J. Friend, Double aperture focusing transducer for controlling microparticle motions in trapezoidal microchannels with surface acoustic waves, Appl. Phys. Lett. 95, 134101 (2009).

[3] A. Lenshof and T. Laurell, Continuous separation of cells and particles in microfluidic systems, Chem. Soc. Rev. 39, 1203 (2010).

[4] X. Ding, Z. Peng, S.-C. S. Lin, M. Geri, S. Li, P. Li, Y. Chen, M. Dao, S. Suresh, and T. J. Huang, Cell separation using tiltedangle standing surface acoustic waves, Proc. Natl. Acad. Sci. USA 111, 12992 (2014).

[5] A. Lenshof, A. Ahmad-Tajudin, K. Jaras, A.-M. Sward-Nilsson, L. Aberg, G. Marko-Varga, J. Malm, H. Lilja, and T. Laurell, Acoustic whole blood plasmapheresis chip for prostate specific antigen microarray diagnostics, Anal. Chem. 81, 6030 (2009).

[6] P. Augustsson, J. T. Karlsen, H.-W. Su, H. Bruus, and J. Voldman, Iso-acoustic focusing of cells for size-insensitive acousto-mechanical phenotyping, Nat. Commun. 7, 11556 (2016).

[7] J. Shi, D. Ahmed, X. Mao, S.-C. S. Lin, A. Lawit, and T. J. Huang, Acoustic tweezers: Patterning cells and microparticles using standing surface acoustic waves (SSAW), Lab Chip 9, 2890 (2009).

[8] A. Riaud, M. Baudoin, O. Bou Matar, L. Becerra, and J.-L. Thomas, Selective Manipulation of Microscopic Particles with Precursor Swirling Rayleigh Waves, Phys. Rev. Appl. 7, 024007 (2017).

[9] Z. Gong and M. Baudoin, Particle Assembly with Synchronized Acoustic Tweezers, Phys. Rev. Appl. 12, 024045 (2019).

[10] D. J. Collins, B. Morahan, J. Garcia-Bustos, C. Doerig, M. Plebanski, and A. Neild, Two-dimensional single-cell patterning with one cell per well driven by surface acoustic waves, Nat. Commun. 6, 8686 (2015).

[11] D. J. Collins, R. O'Rorke, C. Devendran, Z. Ma, J. Han, A. Neild, and Y. Ai, Self-Aligned Acoustofluidic Particle Focusing and Patterning in Microfluidic Channels from Channel-Based Acoustic Waveguides, Phys. Rev. Lett. 120, 074502 (2018).

[12] T. Lilliehorn, U. Simu, M. Nilsson, M. Almqvist, T. Stepinski, T. Laurell, J. Nilsson, and S. Johansson, Trapping of microparticles in the near field of an ultrasonic transducer, Ultrasonics 43, 293 (2005).

[13] M. Evander, L. Johansson, T. Lilliehorn, J. Piskur, M. Lindvall, S. Johansson, M. Almqvist, T. Laurell, and J. Nilsson, Noninvasive acoustic cell trapping in a microfluidic perfusion system for online bioassays, Anal. Chem. 79, 2984 (2007). 
[14] B. Hammarström, M. Evander, H. Barbeau, M. Bruzelius, J. Larsson, T. Laurell, and J. Nillsson, Non-contact acoustic cell trapping in disposable glass capillaries, Lab Chip 10, 2251 (2010).

[15] J. Lei, P. Glynne-Jones, and M. Hill, Acoustic streaming in the transducer plane in ultrasonic particle manipulation devices, Lab Chip 13, 2133 (2013).

[16] P. Mishra, M. Hill, and P. Glynne-Jones, Deformation of red blood cells using acoustic radiation forces, Biomicrofluidics $\mathbf{8}$, 034109 (2014).

[17] I. Gralinski, S. Raymond, T. Alan, and A. Neild, Continuous flow ultrasonic particle trapping in a glass capillary, J. Appl. Phys. 115, 054505 (2014).

[18] M. Grundy, W. Bolek, W. Coakley, and E. Benes, Rapid agglutination testing in an ultrasonic standing wave, J. Immunol. Methods 165, 47 (1993).

[19] M. Tenje, H. Xia, M. Evander, B. Hammarström, A. Tojo, S. Belák, T. Laurell, and N. LeBlanc, Acoustic trapping as a generic noncontact incubation site for multiplex bead-based assays, Anal. Chim. Acta 853, 682 (2014).

[20] B. Hammarström, T. Laurell, and J. Nilsson, Seed particle enabled acoustic trapping of bacteria and nanoparticles in continuous flow systems, Lab Chip 12, 4296 (2012).

[21] M. Evander, O. Gidlof, B. Olde, D. Erlinge, and T. Laurell, Non-contact acoustic capture of microparticles from small plasma volumes, Lab Chip 15, 2588 (2015).

[22] L. V. King, On the acoustic radiation pressure on spheres, Proc. R. Soc. London, Ser. A 147, 212 (1934).

[23] K. Yosioka and Y. Kawasima, Acoustic radiation pressure on a compressible sphere, Acustica 5, 167 (1955).

[24] L. P. Gorkov, On the forces acting on a small particle in an acoustical field in an ideal fluid, Sov. Phys. Dokl. 6, 773 (1962) [On the forces acting on a small particle in an acoustical field in an ideal fluid, Doklady Akademii Nauk SSSR 140, 88 (1961)].

[25] A. A. Doinikov, Acoustic radiation force on a spherical particle in a viscous heat-conducting fluid.1. general formula, J. Acoust. Soc. Am. 101, 713 (1997).

[26] M. Settnes and H. Bruus, Forces acting on a small particle in an acoustical field in a viscous fluid, Phys. Rev. E 85, 016327 (2012).

[27] J. T. Karlsen and H. Bruus, Forces acting on a small particle in an acoustical field in a thermoviscous fluid, Phys. Rev. E 92, 043010 (2015).

[28] Lord Rayleigh, On the circulation of air observed in Kundt's tubes, and on some allied acoustical problems, Philos. Trans. R. Soc. London 175, 1 (1884).

[29] C. Eckart, Vortices and streams caused by sound waves, Phys. Rev. 73, 68 (1948).

[30] J. S. Bach and H. Bruus, Theory of pressure acoustics with viscous boundary layers and streaming in curved elastic cavities, J. Acoust. Soc. Am. 144, 766 (2018).
[31] S. M. Hagsäter, A. Lenshof, P. Skafte-Pedersen, J. P. Kutter, T. Laurell, and H. Bruus, Acoustic resonances in straight micro channels: Beyond the 1D-approximation, Lab Chip 8, 1178 (2008).

[32] M. Evander, A. Lenshof, T. Laurell, and J. Nilsson, Acoustophoresis in wet-etched glass chips, Anal. Chem. 80, 5178 (2008).

[33] D. Carugo, T. Octon, W. Messaoudi, A. L. Fisher, M. Carboni, N. R. Harris, M. Hill, and P. Glynne-Jones, A thin-reflector microfluidic resonator for continuous-flow concentration of microorganisms: A new approach to water quality analysis using acoustofluidics, Lab Chip 14, 3830 (2014).

[34] R. Yuan, J. Lee, H.-W. Su, E. Levy, T. Khudiyev, J. Voldman, and Y. Fink, Microfluidics in structured multimaterial fibers, PNAS 115, E10830 (2018).

[35] P. J. Woodside SM, Bowen BD, Measurement of ultrasonic forces for particle-liquid separations, AIChE J. 43, 1727 (1997).

[36] M. W. H. Ley and H. Bruus, Three-Dimensional Numerical Modeling of Acoustic Trapping in Glass Capillaries, Phys. Rev. Appl. 8, 024020 (2017).

[37] N. R. Skov, J. S. Bach, B. G. Winckelmann, and H. Bruus, 3D modeling of acoustofluidics in a liquid-filled cavity including streaming, viscous boundary layers, surrounding solids, and a piezoelectric transducer, AIMS Mathematics 4, 99 (2019).

[38] J. S. Bach and H. Bruus, Bulk-driven acoustic streaming at resonance in closed microcavities, Phys. Rev. E 100, 023104 (2019).

[39] COMSOL Multiphysics 5.4, http://www.comsol.com.

[40] P. B. Muller, R. Barnkob, M. J. H. Jensen, and H. Bruus, A numerical study of microparticle acoustophoresis driven by acoustic radiation forces and streaming-induced drag forces, Lab Chip 12, 4617 (2012).

[41] P. B. Muller and H. Bruus, Theoretical study of time-dependent, ultrasound-induced acoustic streaming in microchannels, Phys. Rev. E 92, 063018 (2015).

[42] P. B. Muller and H. Bruus, Numerical study of thermoviscous effects in ultrasound-induced acoustic streaming in microchannels, Phys. Rev. E 90, 043016 (2014).

[43] P. Hahn and J. Dual, A numerically efficient damping model for acoustic resonances in microfluidic cavities, Phys. Fluids 27, 062005 (2015).

[44] D. Carugo, D. N. Ankrett, P. Glynne-Jones, L. Capretto, R. J. Boltryk, X. Zhang, P. A. Townsend, and M. Hill, Contrast agent-free sonoporation: The use of an ultrasonic standing wave microfluidic system for the delivery of pharmaceutical agents, Biomicrofluidics 5, 044108 (2011).

[45] P. Reichert, D. Deshmukh, L. Lebovitz, and J. Dual, Thin film piezoelectrics for bulk acoustic wave (BAW) acoustophoresis, Lab Chip 18, 3655 (2018).

[46] Glass Silicon Constraint Substrates, CORNING, New York, USA, http://www.valleydesign.com/Datasheets/Corning\% 20Pyrex\%207740.pdf. 